\title{
Incorporating Robustness Requirements Into Antiwindup Design
}

\author{
Matthew C. Turner, Guido Herrmann, Senior Member, IEEE, and Ian Postlethwaite, Fellow, IEEE
}

\begin{abstract}
This paper treats the problem of synthesizing antiwindup compensators that are able to handle plant uncertainty in addition to controller saturation. The uncertainty considered is of the frequency-weighted additive type, often encountered in linear robust control theory, and representative of a wide variety of uncertainty encountered in practice. The main results show how existing linear matrix inequality based antiwindup synthesis algorithms can be modified to produce compensators that accommodate uncertainty better. Embedded within these results is the ever-present performance-robustness tradeoff. A remarkable feature is that the often criticized internal model control antiwindup solution emerges as an "optimally robust" solution. A simple example demonstrates the effectiveness of the modified algorithms.
\end{abstract}

Index Terms-Antiwindup, constrained control, robust control.

\section{INTRODUCTION}

$\mathbf{M}$ ODEL uncertainty and actuator saturation are two troublesome phenomena frequently encountered by control engineers. The problem of ensuring robustness in the presence of model uncertainty in particular has occupied the control community for many years. Other researchers have devoted much time to the study of actuator saturation, yet, commonly-and remarkably - these problems have been considered in isolation and there are few attempts in the literature to unify some of the results (perhaps the most comprehensive account is given in [1]). It may be argued that actuator saturation could be modelled as a particular type of uncertainty, and therefore it could be handled by standard robust control techniques. However, designs based on this mantra would probably be inherently "low gain" in nature and lead to unduly conservative designs, with restricted small-signal performance.

Conversely, the studies of systems subject to actuator saturation have typically chosen to ignore model uncertainty. This has been the case particularly with the antiwindup community, where the implicit assumption has been that the antiwindup compensated constrained system will inherit the robustness of its unconstrained counterpart. While it appears logical to require nominal linear robustness, this appears to be more of a necessary than sufficient condition to ensure robustness of the overall antiwindup compensated nonlinear system.

The goal of this paper is to develop antiwindup compensators that can be implemented in real systems, which will inevitably contain uncertainty, with some confidence. This paper

Manuscript received November 1, 2005; revised October 6, 2006. Recommended by Associate Editor S. Tarbouriech.

The authors are with the Department of Engineering, University of Leicester, Leicester LE1 7RH, U.K. (e-mail: mct6@le.ac.uk; gh17@le.ac.uk; ixp@le.ac. uk).

Digital Object Identifier 10.1109/TAC.2007.906185 takes as its inspiration the work of [1], which contains a collection of papers that treat the problem of uncertain, saturated systems in a systematic way. However, most of these papers deal with one-step solutions-rather than the two-step antiwindup approach - and a particular type of uncertainty. Specifically, the uncertainty used is normally parametric or state-space uncertainty, generally of the form

$$
\dot{x}=A x+B \operatorname{sat}(u)+\underbrace{\Delta(x, t)}_{\text {uncertainty }} .
$$

Although this type of parametric uncertainty is certainly useful, in practice it is quite limited in scope and is not very useful for capturing unmodelled dynamics, which can be more of an obstacle than their modelled, but uncertain, counterparts.

This paper is based on [2] and continues the work of [3] in a logical way, with more focus on constructive algorithms for antiwindup synthesis. It is important to remark that the motivation for this paper, and also for that of [3], is practical: in our experience in the aerospace and hard-disk control fields, it has really been the unmodelled dynamics that have caused the most difficulty in appropriate controller synthesis and, while present, the parametric uncertainty has played a less prominent role (it is often easily countered by large enough low frequency gain).

The aims of this paper are twofold. First, it aims to bring robustness to the fore in antiwindup compensation, where, except for [4], it has had little prominence. Secondly, it aims to promote the use of a type of uncertainty closer to that often used in practice (and close to that used in linear robust control theory, which has shown itself to be successful in recent years).

The notation used in this paper is standard. In particular, we define the induced $\mathcal{L}_{2}$ norm, or finite $\mathcal{L}_{2}$ gain, of an operator $\mathcal{H}$ as $\|\mathcal{H}\|_{i, 2}:=\sup _{0 \neq x \in \mathcal{L}_{2}}\left(\|\mathcal{H} x\|_{2}\right) /\left(\|x\|_{2}\right)$, where $\|x\|_{2}=$ $\sqrt{\int_{0}^{\infty}\|x\|^{2} d t}$ is the $\mathcal{L}_{2}$ norm of the vector $x(t)$ and $\|x\|$ is its Euclidean norm. The $\mathcal{H}_{\infty}$ norm for a (stable) linear operator $P$ is defined as $\|P\|_{\infty}:=\sup _{\omega} \bar{\sigma}[P(j \omega)]$, where $\bar{\sigma}(\cdot)$ denotes the maximum singular value and $P(j \omega)$ is the frequency response matrix associated with the linear operator $P$. We do not explicitly distinguish between a linear operator and its transfer function. Equivalently, the $\mathcal{H}_{\infty}$ norm may be defined as $\|P\|_{\infty}=$ $\|P\|_{i, 2}$. We denote an asymptotically stable, rational transfer function/linear operator $P$ by $P \in \mathcal{R} \mathcal{H}_{\infty}$. A feedback system is said to be "well posed" when there exists a unique solution to the feedback equations. For linear systems, a simple condition for this can be found in, for example, [5].

\section{A GENERAL ANTIWINDUP FRAMEWORK}

It is convenient to study the robustness of antiwindup (AW) compensators in a framework similar to that proposed in [6] (see 


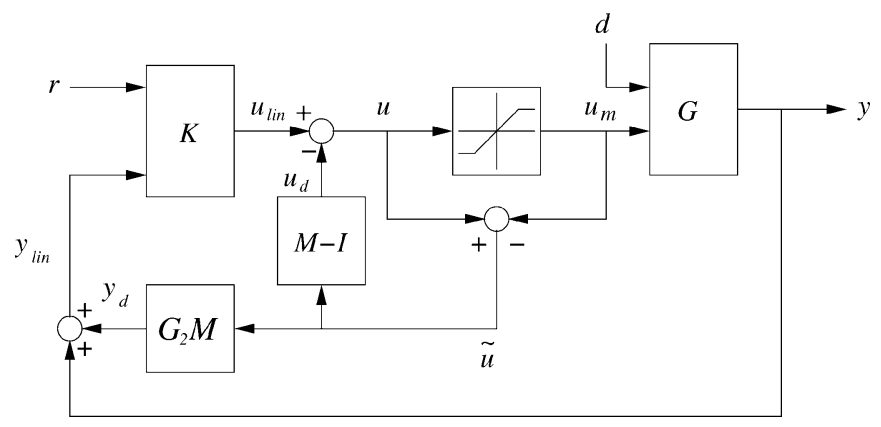

Fig. 1. Conditioning with $M(s)$.

also [7]). This scheme is shown in Fig. 1, where $G(s)$ is the transfer function matrix of the plant and $K(s)$ is the transfer function matrix of the controller, which have state-space realizations

$$
\begin{aligned}
G(s) & \sim\left[\begin{array}{l|ll}
A_{p} & B_{p d} & B_{p} \\
\hline C_{p} & D_{p d} & D_{p}
\end{array}\right] \\
K(s) & \sim\left[\begin{array}{l|ll}
A_{c} & B_{c r} & B_{c} \\
\hline C_{c} & D_{c r} & D_{c}
\end{array}\right]
\end{aligned}
$$

For the analysis here, it is convenient to split the plant function as $G(s)=\left[G_{1}(s) G_{2}(s)\right]$, where $G_{1}(s) \sim\left(A_{p}, B_{p d}, C_{p}, D_{p d}\right)$ is the transfer function from the disturbance $d(t) \in \mathbb{R}^{n_{d}}$ to the measured output $y(t) \in \mathbb{R}^{p}$ and $G_{2}(s) \sim\left(A_{p}, B_{p}, C_{p}, D_{p}\right)$ is the transfer function from the plant input $u_{m}(t) \in \mathbb{R}^{m}$ to the output. In operator form, we have

$$
y=\left[\begin{array}{ll}
G_{1} & G_{2}
\end{array}\right]\left[\begin{array}{c}
d \\
u_{m}
\end{array}\right] .
$$

Similarly, the controller is partitioned as $K(s)=$ $\left[K_{1}(s) K_{2}(s)\right]$, where $K_{1}(s) \sim\left(A_{c}, B_{c r}, C_{c}, D_{c r}\right)$ represents the feedforward part of the controller due to the reference $r(t) \in \mathbb{R}^{n_{r}}$ and $K_{2} \sim\left(A_{c}, B_{c}, C_{c}, D_{c}\right)$ represents the feedback part of the controller due to $y_{\text {lin }}(t)$. Thus the "linear" control signal is determined by

$$
u_{\text {lin }}=\left[\begin{array}{ll}
K_{1} & K_{2}
\end{array}\right]\left[\begin{array}{c}
r \\
y_{\text {lin }}
\end{array}\right] \text {. }
$$

Such plant and controller partitions are not necessary but they do clarify the fact that the disturbance feedforward part of the plant and the reference feedforward part of the controller play no role in the antiwindup performance or robustness problem, as defined here. Obviously, this partition assumes that $K_{1}(s)$ contains only the same unstable (normally integral modes) as $K_{2}(s)$ to ensure stability of the system. We would normally assume similarly that $G_{1}(s)$ contains only the same unstable modes as $G_{2}(s)$, but for reasons of global stability, we make the stronger assumption that $G(s) \in \mathcal{R} \mathcal{H}_{\infty}$.

The control signal produced by the controller and, if active, the antiwindup compensator is $u(t) \in \mathbb{R}^{m}$, which may differ to the actual plant input $u_{m}(t)=\operatorname{sat}(u(t))$ due to the saturation nonlinearity between the two signals. The antiwindup compensator is described by the transfer function matrix $[(M(s)-$ $\left.I)^{\prime}\left(G_{2}(s) M(s)\right)^{\prime}\right]^{\prime}$ and features a copy of the known feedback portion of the plant $G_{2}(s)$ and a free parameter- $-M(s) \in$

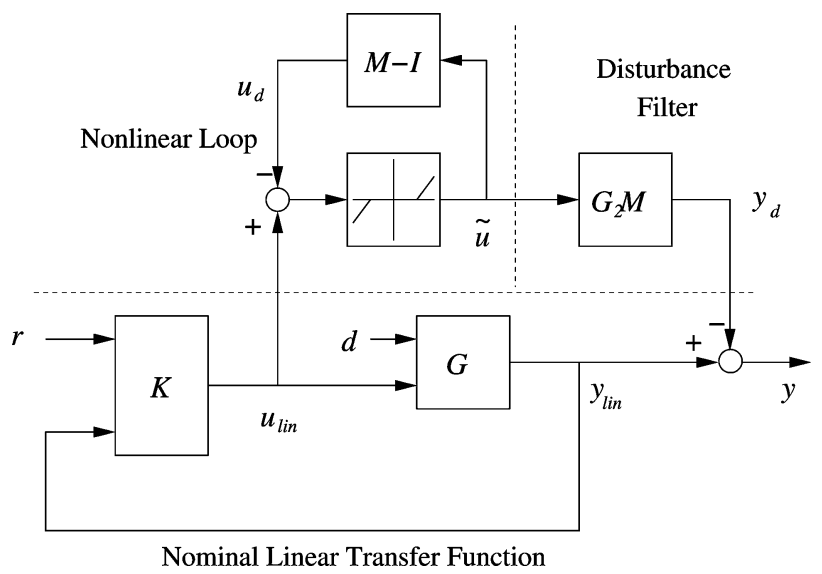

Fig. 2. Equivalent representation of Fig. 1.

$\mathcal{R} \mathcal{H}_{\infty}$. It generates two signals $u_{d}(t) \in \mathbb{R}^{m}$, which are fed to the controller output, and a further signal $y_{d}(t) \in \mathbb{R}^{p}$, which is fed to the controller input, i.e.,

$$
\left[\begin{array}{l}
u_{d} \\
y_{d}
\end{array}\right]=\left[\begin{array}{c}
M-I \\
G_{2} M
\end{array}\right] \tilde{u} .
$$

The antiwindup compensator is driven by the difference between the controller output and plant input $\tilde{u}=u-u_{m}$ and thus, assuming zero initial conditions, remains inactive until saturation occurs. When saturation does occur, the antiwindup compensator becomes active and thus the controller output and one of the controller inputs are modified to

$$
\begin{aligned}
u(t) & =u_{\operatorname{lin}}(t)-u_{d}(t) \\
y_{\operatorname{lin}}(t) & =y(t)+y_{d}(t) .
\end{aligned}
$$

When $u(t) \equiv u_{m}(t)$ and, assuming zero initial conditions for the antiwindup compensator, $u_{d}(t) \equiv 0, y_{d}(t) \equiv 0$, we say the system exhibits nominal behavior. Parameterizing the antiwindup compensator in the above way allows Fig. 1 to be redrawn as the equivalent scheme shown in Fig. 2, where we have used the relationship

$$
\operatorname{sat}(u)=u-\operatorname{Dz}(u)
$$

where

$$
\operatorname{sat}(u)=\left[\begin{array}{c}
\operatorname{sat}_{1}\left(u_{1}\right) \\
\vdots \\
\operatorname{sat}_{m}\left(u_{m}\right)
\end{array}\right] \quad \operatorname{Dz}(u)=\left[\begin{array}{c}
\operatorname{Dz}_{1}\left(u_{1}\right) \\
\vdots \\
\operatorname{Dz}_{m}\left(u_{m}\right)
\end{array}\right]
$$

and $\operatorname{sat}_{i}\left(u_{i}\right)=\operatorname{sign}\left(u_{i}\right) \min \left(\left|u_{i}\right|, \bar{u}_{i}\right) \forall i$ and $\operatorname{Dz}_{i}\left(u_{i}\right)=$ $\operatorname{sign}\left(u_{i}\right) \max \left(0,\left|u_{i}\right|-\bar{u}_{i}\right) \forall i$. Also $\bar{u}_{i}>0 \forall i \in\{1, \ldots, m\}$.

As noted in [7], the architecture of the AW scheme in Fig. 2 reveals a more lucid representation of the AW problem. In particular, under the logical assumption that the nominal, unsaturated, feedback combination of $K(s)$ and $G(s)$ is asymptotically stable, then providing that $G_{2}(s) \in \mathcal{R} \mathcal{H}_{\infty}$, the nonlinear stability problem in Fig. 1 is simply translated to picking $M(s) \in \mathcal{R H}_{\infty}$ such that the nonlinear loop in Fig. 2 is asymptotically stable. Moreover, from Fig. 2, it can be seen that the 


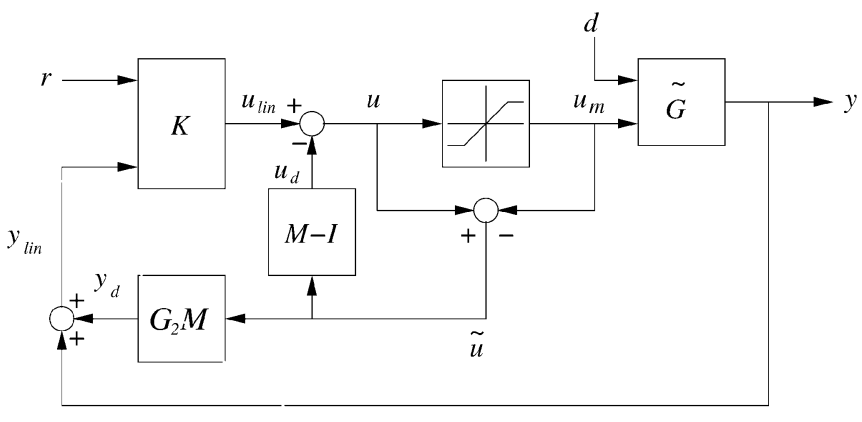

Fig. 3. Antiwindup with uncertainty.

performance of the antiwindup compensator is intimately related to the mapping $\mathcal{T}_{p}: u_{\text {lin }} \mapsto y_{d}$ : if the norm of this mapping is small, then the antiwindup compensator is successful at keeping performance close to linear (which we assume is the desired performance). In [8], it was shown how the $\mathcal{L}_{2}$ gain of $\mathcal{T}_{p}$ could be minimized using a system of linear matrix inequalities and, furthermore, how $M(s)$ could be chosen such that it corresponded to static or low-order antiwindup compensators (discrete and sampled-data versions of this can be found in [9]-[11]). It was demonstrated, using suitable examples, in [8] that direct minimization of $\mathcal{T}_{p}$ was central to good antiwindup performance and compensators designed according to the ideas in [8] seemed to perform at least as well as, and often better than, most other antiwindup compensators. Furthermore, in [7], it was shown that most antiwindup schemes can be interpreted as certain choices of $M(s) \in \mathcal{R H}_{\infty}$, and therefore schemes such as the Hanus conditioning scheme [12], the high gain approach [13], [14], and such like can be analyzed in terms of Fig. 1, or, equivalently, Fig. 2.

\section{A. Plant Uncertainty}

Consider the configuration in Fig. 3, where $\tilde{G}$ is the true plant given by $\tilde{G}(s)=\left[G_{1}(s) G_{2}(s)+W_{2}(s) \Delta_{G}(s)\right]$, where $G(s)=$ $\left[G_{1}(s) G_{2}(s)\right]$ is the model of the plant with which we work and $W_{2}(s) \Delta_{G}(s)$ is additive uncertainty to the feedback part. ${ }^{1} \mathrm{We}$ assume that the uncertainty $W_{2}(s) \Delta_{G}(s)$ comprises a "known part" $W_{2}(s) \in \mathcal{R H}_{\infty}$, which typically is a crude model of its frequency response, and an unknown part $\Delta_{G}(s) \in \boldsymbol{\Delta}$, where

$$
\Delta=\left\{\Delta \in \mathcal{R} \mathcal{H}_{\infty}:\|\Delta\|_{\infty}<\frac{1}{\delta}\right\} .
$$

This is a reasonably generic type of uncertainty, although we do not use the representation $W_{2} \Delta_{G} W_{1}$, as this complicates the small gain argument we use later in this paper. Other types of uncertainty such as output-multiplicative uncertainty, where $\tilde{G}(s)=\left(I+\Delta_{o}(s)\right) G_{2}(s)$, and input-multiplicative uncertainty $\tilde{G}(s)=G_{2}(s)\left(I+\Delta_{i}(s)\right)$ could be used instead, of course. However, it is easy to see that both these uncertainties can be captured by additive uncertainty $\left(W_{2} \Delta_{G}=G_{2} \Delta_{i}\right.$ or $W_{2} \Delta_{G}=$ $\Delta_{o} G_{2}$ ), although the converse is not always true (unless $G_{2}$ is invertible), so we prefer to work with additive uncertainty.

\footnotetext{
${ }^{1}$ It is likely that there will also be a perturbation of the disturbance feedforward portion of the plant $G_{1}$, although this will have no bearing on global stability results, so for simplicity we do not consider it.
}

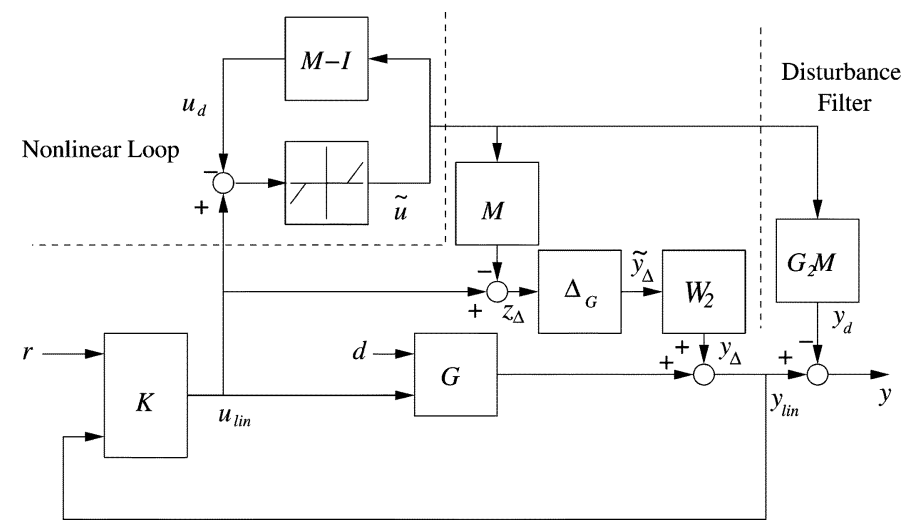

Fig. 4. Equivalent representation of Fig. 3.

When uncertainty is present in the system, the appealing decoupled structure of the original scheme is lost. Fig. 4 shows an equivalent representation of Fig. 3. All signals have the same meaning as before, and the additional signals $\tilde{y}_{\Delta}:=\Delta_{G}\left(u_{\text {lin }}-\right.$ $M \tilde{u})$ and $y_{\Delta}:=W_{2} \tilde{y}_{\Delta}$ represent the effect of the uncertainty on the "linear loop." It is important to mention that the term $W_{2} \Delta_{G} M: \tilde{u} \mapsto y_{\Delta}$ destroys the decoupling of the linear system and nonlinear loop; the "linear loop" is no longer linear.

\section{B. Assumptions}

We make the following assumptions.

1) The open-loop plant $G(s)$ is asymptotically stable. In order to obtain global finite-gain stability results for constrained input systems, this assumption is vital.

2) The (linear) uncertainty $W_{2}(s) \Delta_{G}(s)$ is asymptotically stable. This mirrors the case in standard $\mathcal{H}_{\infty}$ control theory where the perturbations are assumed stable. This greatly simplifies the work and is necessary for the small-gain approach we take.

3) The nominal linear closed-loop system is robustly asymptotically stable and well posed. By this, we mean that, when the saturation nonlinearity is replaced by the identity operator, the closed-loop system is stable and furthermore can tolerate a certain amount of uncertainty $\left(\left\|\Delta_{G}\right\|_{\infty}<(1 / \delta)\right.$, where $\left.\delta=\left\|\left(I-K_{2} G_{2}\right)^{-1} K_{2} W_{2}\right\|_{\infty}\right)$ before becoming unstable. This essentially amounts to assuming that the design of the linear controller $K(s)$ is "good" in the sense that it robustly stabilizes the system. We also assume that the nominal linear closed loop is well posed. These two assumptions can be summarized as

$$
\begin{aligned}
Z(s) & =\left[\begin{array}{cc}
I & -K_{2}(s) \\
-\left(G_{2}(s)+W_{2}(s) \Delta_{G}(s)\right) & I
\end{array}\right]^{-1} \\
& \in \mathcal{R} \mathcal{H}^{\infty} \quad \forall \Delta_{G}(s) \in \boldsymbol{\Delta}
\end{aligned}
$$

and $Z(\infty)$ exists. Note that the uncertainty model $W_{2} \Delta_{G}$ is somewhat less restrictive than the $\Delta_{G}$ used in [2], as it does not require the transfer function $\left(I-K_{2} G_{2}\right)^{-1} K_{2}$ to be small across all frequencies.

4) The nominal linear closed loop yields desirable performance. As is common in the antiwindup literature, it is assumed that the linear closed loop yields desired perfor- 
mance and the performance of the antiwindup compensator can be measured against the deterioration of this performance when the control signals saturate. This is related to the foregoing point in the sense that we also assume that the linear closed-loop yields desirable robustness properties and therefore the performance of the antiwindup compensator can also be assessed against its preservation of the linear system's robustness properties.

On the basis of these assumptions, three features are evident from Fig. 4.

1) If $\Delta_{G}$ is small in some sense, then the robustness of the antiwindup scheme is similar to that of the nominal, unconstrained linear system (via a small-gain argument).

2) If the mapping from $u_{\text {lin }} \mapsto M \tilde{u}$ is small, again, the robustness of the antiwindup system is similar to that of the nominal linear system (again using a small-gain argument).

3) The robustness of the system with antiwindup compensation can never be better than the robustness of the linear system. Denoting the map from $u_{\text {lin }}$ to $\tilde{u}$ as $\mathcal{F}(\cdot): \mathbb{R}^{m} \mapsto \mathbb{R}^{m}$ (the nonlinear loop), the "modified" uncertainty can be represented as $\tilde{\Delta}_{G}: u_{\text {lin }} \mapsto \tilde{y}_{\Delta}$, where $\tilde{\Delta}_{G}$ is given by the expression $\tilde{y}_{\Delta}=\tilde{\Delta}_{G}\left(u_{\text {lin }}\right):=\Delta_{G}\left(u_{\text {lin }}-M \mathcal{F}\left(u_{\text {lin }}\right)\right)$. From this it can be noted (see the Appendix) that $\left\|\Delta_{G}\right\|_{\infty}=\left\|\Delta_{G}\right\|_{i, 2} \leq\left\|\tilde{\Delta}_{G}\right\|_{i, 2}$ and therefore retention of the linear system's robustness properties can be considered as an optimal property. This will be discussed in more detail later.

In what follows, we shall make these notions of robustness more precise, and algorithms will be proposed that ensure our system with antiwindup is asymptotically stable for all $\Delta_{G}$ in the set

$$
\boldsymbol{\Delta}_{\gamma \delta}:=\left\{\Delta_{G} \in \mathcal{R} \mathcal{H}_{\infty}:\left\|\Delta_{G}\right\|_{\infty}<\frac{1}{\gamma \delta}\right\} .
$$

In particular we shall be interested in minimizing $\gamma$ to ensure that the uncertainty set $\boldsymbol{\Delta}_{\gamma \delta}$ is as large as possible. Note that, as indicated already, the robustness of the antiwindup scheme cannot be better than the nominal linear robustness, and it will be seen that $\gamma \geq 1$, where $\gamma$ is an $\mathcal{L}_{2}$ gain parameter introduced later.

\section{SPECIAL CASE: IMC ANTIWINDUP}

Before we explore the consequences of uncertainty in antiwindup further, it is interesting to consider a special case: the much-maligned internal model control (IMC) antiwindup scheme. IMC antiwindup was introduced in [15] as an antiwindup methodology, but many examples in the literature have shown it to be poor (e.g., [16]). This can be easily seen by viewing IMC antiwindup in Fig. 2: to obtain IMC antiwindup, we simply choose $M=I$. The nonlinear "loop" becomes simply the dead-zone operator $\mathrm{Dz}(\cdot)$ and the disturbance filter becomes the open-loop plant $G_{2}(s)$. Hence the IMC performance will be poor if the open-loop plant has slow, lightly damped poles or nonminimum phase zeros.

As is often the case in linear robust control theory, there is a tradeoff between performance and robustness, and this seems

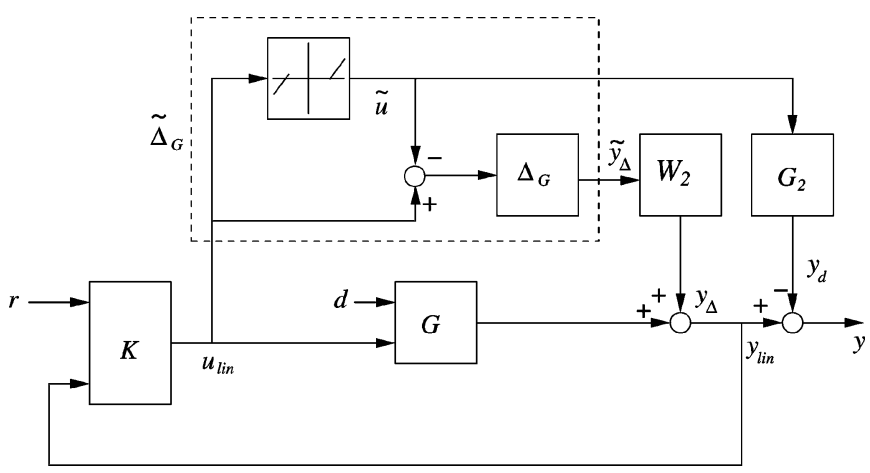

Fig. 5. IMC antiwindup with uncertainty.

to extend to antiwindup compensation. Consider uncertain antiwindup in Fig. 4 and choose $M=I$; then again the nonlinear loop degenerates to the dead-zone function and the troublesome term, which destroys the decoupling of the linear and nonlinear parts of the system, simply becomes the uncertainty $W_{2} \Delta_{G}$. For convenience, this scenario is redrawn in Fig. 5. For consistency, we have retained the notation $y_{\text {lin }}$ and $u_{\text {lin }}$, although it should be understood that these signals are no longer generated by a purely linear system.

Now, nominally, assuming no saturation, simple small gain analysis shows that we have stability robustness against all input additive uncertainty

$$
\left\|\Delta_{G}\right\|_{\infty}<\frac{1}{\delta}
$$

where $\left\|\left(I-K_{2} G_{2}\right)^{-1} K_{2} W_{2}\right\|_{\infty}:=\delta$. Similarly, a small gain analysis of Fig. 5 reveals that stability is maintained providing that the "modified" nonlinear uncertainty $\tilde{\Delta}_{G}: u_{\text {lin }} \mapsto \tilde{y}_{\Delta}$ satisfies

$$
\left\|\tilde{\Delta}_{G}\right\|_{i, 2}<\frac{1}{\delta}
$$

But as

$$
\begin{aligned}
\left\|\tilde{\Delta}_{G}\right\|_{i, 2} & \leq\left\|\Delta_{G}\right\|_{\infty}\|I-D z(\cdot)\|_{i, 2} \\
& =\left\|\Delta_{G}\right\|_{\infty}\|\operatorname{sat}(\cdot)\|_{i, 2} \\
& =\left\|\Delta_{G}\right\|_{\infty}
\end{aligned}
$$

stability robustness is ensured for all uncertainty satisfying (11), that is, all uncertainty within the ball $\Delta$. However, as $\left\|\Delta_{G}\right\|_{\infty} \leq$ $\left\|\tilde{\Delta}_{G}\right\|_{i, 2}$, then $\left\|\tilde{\Delta}_{G}\right\|_{i, 2}=\left\|\Delta_{G}\right\|_{\infty}$. In other words, the IMC antiwindup scheme is guaranteed to be robustly stable for the same class of additive uncertainties as the nominal linear system. Recall that it is not possible for an antiwindup scheme to be more robust than the nominal linear system because much of the antiwindup scheme's time is spent operating as a linear system (see Appendix I). So, for the uncertainty class considered here, retention of the linear system's robustness properties is optimal. Hence, although IMC schemes can be criticized for their performance, they are in fact optimally robust as far as stability is concerned. A similar observation was made in [17]. 


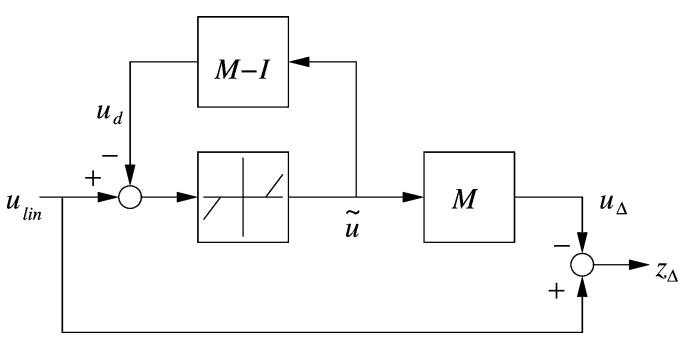

Fig. 6. Robustness optimization for general antiwindup schemes: graphical representation of $\mathcal{T}_{r}$.

\section{General CASE}

\section{A. A Stability Robustness Criterion}

From Fig. 4, we have that

$$
\begin{aligned}
y_{\text {lin }} & =G_{1} d+G_{2} u_{\operatorname{lin}}+W_{2} \Delta_{G}\left[u_{\operatorname{lin}}-M \mathcal{F}\left(u_{\text {lin }}\right)\right] \\
& =G_{1} d+G_{2} u_{\operatorname{lin}}+W_{2} \tilde{\Delta}_{G}\left(u_{\operatorname{lin}}\right)
\end{aligned}
$$

where, as before, $\mathcal{F}\left(u_{\text {lin }}\right)$ is the map from $u_{\text {lin }}$ to $\tilde{u}$. Carrying out a small gain analysis, we see that the system is robust against all additive perturbations such that

$$
\left\|\tilde{\Delta}_{G}\right\|_{i, 2}=\left\|\Delta_{G}\left[I-M \mathcal{F}\left(u_{\operatorname{lin}}\right)\right]\right\|_{i, 2}<\frac{1}{\delta} .
$$

So nominal robustness is retained if $\left\|\left[I-M \mathcal{F}\left(u_{\text {lin }}\right)\right]\right\|_{i, 2} \leq 1$. However, as $\mathcal{F}\left(u_{\text {lin }}\right)=0$ around $u_{\text {lin }}=0$ (as it contains the dead zone), $\left\|\left[I-M \mathcal{F}\left(u_{\text {lin }}\right)\right]\right\|_{i, 2}$ can never be strictly less than unity. Again this conclusion coincides with our prior discussion, as we could not expect an antiwindup scheme to yield greater robustness margins than the linear system upon which it is constructed. This also serves as justification for the IMC scheme, although it is unlikely to be the unique compensator that achieves this optimality.

\section{B. Stability Robustness Optimization}

In order to preserve as much robustness as possible, we would like to minimize $\left\|\mathcal{T}_{r}\right\|_{i, 2}:=\left\|\left[I-M \mathcal{F}\left(u_{\text {lin }}\right)\right]\right\|_{i, 2}$, preferably making it as close to unity as possible. Diagrammatically, this can be shown as Fig. 6, where we want to minimize the $\mathcal{L}_{2}$ gain from $u_{\text {lin }}$ to $z_{\Delta}$, where $z_{\Delta}:=\mathcal{T}_{r}\left(u_{\text {lin }}\right)$ is the signal feeding into the uncertainty, as shown in Fig. 4. If we can guarantee that $\left\|\mathcal{T}_{r}\right\|_{i, 2}<\gamma$, small gain analysis of Fig. 4 then shows that the system will be asymptotically stable providing that the nonlinear loop $\mathcal{F}(\cdot)$ is asymptotically stable $G_{2}(s) M(s) \in$ $\mathcal{R H}_{\infty}$ and the uncertainty $\Delta_{G}(s) \in \boldsymbol{\Delta}_{\gamma \delta}$. Note that when $\gamma=1, \boldsymbol{\Delta}_{\gamma \delta}=\boldsymbol{\Delta}$ and robust stability equal to that of the linear system is obtained. Thus the goal of our optimization procedure should be to minimize $\gamma$, making it as close to unity as possible. As mentioned in [8], this optimization is typically a difficult optimization problem to solve, so instead we shall be content to ensure a certain $\mathcal{L}_{2}$ gain bound holds for the map $\mathcal{T}_{r}$.

We shall consider robustness optimization using full-order antiwindup compensators. As suggested in [7], we choose $M(s)$ to be part of a right-coprime factorization of $G_{2}(s)=N(s) M^{-1}(s)$ (this in fact is a dual result to that of [18], where antiwindup is described as a left coprime factorization of the controller) and attempt to choose a particular factorization such that robustness is optimized. Given the plant realization $G_{2}(s) \sim\left(A_{p}, B_{p}, C_{p}, D_{p}\right)$, all full-order right coprime factorizations (up to multiplication by an invertible matrix) can be described [7] as

$$
\left[\begin{array}{c}
M(s) \\
N(s)
\end{array}\right] \sim\left[\begin{array}{c|c}
A_{p}+B_{p} F & B_{p} \\
F & I \\
C_{p}+D_{p} F & D_{p}
\end{array}\right]
$$

where $F$ is chosen such that $A_{p}+B_{p} F$ is a Hurwitz matrix. This choice of coprime factorization prevents implicit equations (algebraic loops) from appearing in the nonlinear loop (as depicted in Figs. 2 and 4, for example) as $M(s)-I$ is strictly proper. The coprime factor representation implies that $G_{2}(s) M(s)=N(s) \in \mathcal{R} \mathcal{H}_{\infty}$, so, in order to guarantee robust stability for all $\Delta_{G} \in \Delta$, it suffices to ensure that $\left\|\mathcal{T}_{r}\right\|_{i, 2}<\gamma$ (for $\gamma \leq 1$ ) and the nonlinear loop $\mathcal{F}(\cdot)$ is well posed. This will indeed be the case if the following inequality holds for sufficiently small $\gamma$ :

$$
J=\frac{d}{d t} x^{\prime} P x+\left\|z_{\Delta}\right\|^{2}-\gamma^{2}\left\|u_{\text {lin }}\right\|^{2}<0 \quad \forall\left[\begin{array}{ll}
x^{\prime} & u_{\text {lin }}^{\prime}
\end{array}\right]^{\prime} \neq 0
$$

where $x$ is the state vector associated with the realization of $\left[M(s)-I^{\prime}, M(s)^{\prime}\right]^{\prime}$ and $P$ is some positive definite symmetric matrix. As shown in, for example, [19] and [8], this ensures that the $\mathcal{L}_{2}$ gain from $u_{\text {lin }}$ to $z_{\Delta}$ is less than $\gamma$ and that the system in Fig. 6 is asymptotically stable.

As the dead-zone nonlinearity belongs to Sector $[0, I]$ (see [20, ch. 10]), we make use of the inequality

$$
2 \tilde{u}^{\prime} X\left(u_{\operatorname{lin}}-u_{d}-\tilde{u}\right) \geq 0
$$

where the "multiplier" matrix $X:=\operatorname{diag}\left(\chi_{1}, \ldots, \chi_{m}\right)>0$ is chosen diagonal as (18) is in fact $m$ scalar inequalities-see, for example, [21, ch. 12]. This can be adjoined to (17) using the $\mathrm{S}$-procedure to obtain the modified function

$$
\tilde{J}=\frac{d}{d t} x^{\prime} P x+\left\|z_{\Delta}\right\|^{2}-\gamma^{2}\left\|u_{\operatorname{lin}}\right\|^{2}+2 \tilde{u}^{\prime} X\left(u_{\operatorname{lin}}-u_{d}-\tilde{u}\right) .
$$

If $\tilde{J}<0 \forall\left[\begin{array}{lll}x^{\prime} & \tilde{u}^{\prime} & u_{\text {lin }}^{\prime}\end{array}\right] \neq 0$, this implies that $J<0$. Evaluating $\tilde{J}$ in a similar manner to [8] (see also [9] and [10] for the discrete and sampled data cases) yields the following linear matrix inequality (LMI):

$$
\begin{aligned}
& {\left[\begin{array}{cccc}
Q A_{p}^{\prime}+A_{p} Q+L^{\prime} B_{p}^{\prime}+B_{p} L & B_{p} U-L^{\prime} & 0 & L^{\prime} \\
\star & -2 U & I & U \\
\star & \star & -\gamma I & -I \\
\star & \star & \star & -\gamma I
\end{array}\right]} \\
& <0
\end{aligned}
$$

in the variables $Q:=P^{-1}>0, X^{-1}=: \quad U=$ $\operatorname{diag}\left(\nu_{1}, \ldots, \nu_{m}\right)>0, L, \gamma>0$.

Satisfaction of this LMI means that (19) is satisfied and hence that the $\mathcal{L}_{2}$ gain from $u_{\text {lin }}$ to $z_{\Delta}$ is less than $\gamma$ and a suitable choice of $F$ is given by $F=L Q^{-1}$. A small gain analysis of Fig. 4 reveals that, as $\left\|\mathcal{T}_{r}\right\|_{i, 2}<\gamma$ and 
$\left\|\left(I-K_{2} G_{2}\right)^{-1} K_{2} W_{2}\right\|_{\infty}=\delta$, then our system will be asymptotically stable for all $\Delta_{G} \in \boldsymbol{\Delta}_{\gamma \delta}$.

Note from the lower right $2 \times 2$ block of the LMI (20)

$$
\left[\begin{array}{cc}
-\gamma I & -I \\
\star & -\gamma I
\end{array}\right]
$$

that, as anticipated earlier, the $\mathcal{L}_{2}$ gain can be no less than unity, which is achieved for the IMC scheme.

\section{Optimization for Robustness and Performance}

The primary goal of antiwindup compensation is to provide performance improvement during saturation, but optimizing the LMI (20) alone does not guarantee this. Indeed, there is little point in optimizing (20) when an optimal solution can be found by inspection as the IMC antiwindup solution. The real use of (20) and the arguments of the previous subsection is to use them in conjunction with performance optimization, the goal being to optimize performance and robustness together, although there will often be a tradeoff.

In [8], it was argued that $\mathcal{T}_{p}$, the map from $u_{\text {lin }}$ to $y_{d}$ was central to the "true goal" of antiwindup compensation: if the induced norm of this operator was minimized, the deviation of the system's nonlinear behavior during and after saturation would be minimized. This paper [8] solved this problem with $\Delta_{G}=0$, in the $\mathcal{L}_{2}$ sense, for static and low-order compensators (similar treatments for discrete-time AW compensators were given in [9] and [10]).

More realistically, we would really like to optimize some weighted combination of $\mathcal{T}_{p}$ and $\mathcal{T}_{r}$. This can be accomplished by ensuring that

$$
\left\|\begin{array}{c}
W_{p}^{\frac{1}{2}} y_{d} \\
W_{r}^{\frac{1}{2}} z_{\Delta}
\end{array}\right\|_{2}<\gamma\left\|u_{\operatorname{lin}}\right\|_{2}
$$

where $W_{p}$ and $W_{r}$ are weighting matrices that reflect the relative importance of performance and robustness, respectively, and are chosen by the designer. Taking advantage of the sector bound (18), we are sure that (21) holds if

$$
\begin{aligned}
J=\frac{d}{d t} x^{\prime} P x & +\left\|\begin{array}{c}
W_{p}^{\frac{1}{2}} y_{d} \\
W_{r}^{\frac{1}{2}} z_{\Delta}
\end{array}\right\|^{2}-\gamma^{2}\left\|u_{\text {lin }}\right\|^{2} \\
& +2 \tilde{u}^{\prime} X(u-\tilde{u})<0 \quad \forall\left[\begin{array}{lll}
x^{\prime} & \tilde{u}^{\prime} & u_{\text {lin }}^{\prime}
\end{array}\right]^{\prime} \neq 0 .
\end{aligned}
$$

As before, this inequality holds if the LMI as shown in (23) at the bottom of the page (in the variables $Q>0, U=$ $\left.\operatorname{diag}\left(\nu_{1}, \ldots, \nu_{m}\right)>0, L, \gamma>0\right)$ is satisfied The derivation of this LMI is carried out in a similar way to that of the previous section, in the spirit of that done in [8]. Again the matrix $F$ is recovered through $F=L Q^{-1}$. The presence of the weighting matrix $W_{r}$ ensures that $\left\|\mathcal{T}_{r}\right\|_{i, 2}<\gamma / \sqrt{\underline{\sigma}\left(W_{r}\right)}$ and hence that asymptotic stability of Fig. 4 (and hence Fig. 3) is guaranteed for all perturbations $\Delta_{G}(s) \in \boldsymbol{\Delta}_{\tilde{\gamma} \delta}$, where

$$
\boldsymbol{\Delta}_{\tilde{\gamma} \delta}:=\left\{\Delta_{G} \in \mathcal{R} \mathcal{H}_{\infty}:\left\|\Delta_{G}\right\|_{\infty}<\frac{1}{\tilde{\gamma} \delta}\right\}
$$

and $\tilde{\gamma}:=\gamma / \sqrt{\underline{\sigma}\left(W_{r}\right)}$. Obviously, if $W_{r}=I, \boldsymbol{\Delta}_{\tilde{\gamma} \delta}=\boldsymbol{\Delta}_{\gamma \delta}$.

Remark 1: Note that satisfaction of LMI (23) ensures that, with no uncertainty $\Delta_{G}=0$, the nonlinear loop in Fig. 4 is globally asymptotically stable and $\mathcal{T}_{p}$ is finite $\mathcal{L}_{2}$ gain stable. Thus as Figs. 4 and 3 are mathematically equivalent, this ensures global asymptotic and finite $\mathcal{L}_{2}$ gain of the overall saturated system. Similarly, when $\Delta_{G} \neq 0$, satisfaction of the LMI (23) ensures that the system in Fig. 3 is globally asymptotically stable with finite $\mathcal{L}_{2}$ gain providing that $\left\|\Delta_{G}\right\|_{\infty} \leq\left(\sqrt{\underline{\sigma}\left(W_{r}\right)}\right) /(\gamma \delta)$. Thus providing $\tilde{\gamma} \approx 1$ (for example, if $W_{r}=I$ and $\gamma \approx 1$ ), the saturated system with $\mathrm{AW}$ is robust against a similar class of uncertainties $(\boldsymbol{\Delta})$ to the nominal linear system.

Remark 2: Another advantage of using LMI (23) to synthesize full-order compensators is that it tends to prevent fast poles appearing in the compensator dynamics. If a robustness weight $\left(W_{r}\right)$ were not included in the optimization - or if $W_{r}$ were only chosen small - the poles of the antiwindup compensator would tend to be rather fast, lying far to the left of the imaginary axis. Obviously this would require a very high sampling frequency for implementation, which is not always possible in practice. However, when simultaneously optimizing performance and robustness using (23), the poles are placed in regions more comparable to that of the controller. To see this, note that LMI (23) equivalent to the matrix inequality (25), as shown at the bottom of the next page, is satisfied.

A necessary condition for this to hold is obtained from the (1, 1) term, which with $L=F Q$ is

$$
\begin{aligned}
Q\left(A_{p}+B_{p} F\right)^{\prime}+\left(A_{p}+\right. & \left.B_{p} F\right) Q \\
& +\gamma^{-1} Q F^{\prime} B_{p}^{\prime} W_{r} B_{p} F Q<0 .
\end{aligned}
$$

Note that when $W_{r}=0$ (i.e., robustness is not included), the matrix $F$ is not constrained in magnitude, but when $W_{r} \neq 0$, it must be sufficiently "small" to ensure that (26) holds. This feature is reminiscent of solving "singular" $\mathcal{H}_{\infty}$ problems with LMIs, where poles tend to get placed far from the imaginary axis. Of course in the Riccati-based methods, these singular problems are not solvable, which normally prevents the appearance of these large poles.

$$
\left[\begin{array}{ccccc}
Q A_{p}^{\prime}+A_{p} Q+L^{\prime} B_{p}^{\prime}+B_{p} L & B_{p} U-L^{\prime} & 0 & Q C_{p}^{\prime}+L^{\prime} D_{p}^{\prime} & L^{\prime} \\
\star & -2 U & I & U D_{p}^{\prime} & U \\
\star & \star & -\gamma I & 0 & -I \\
\star & \star & \star & -\gamma W_{p}^{-1} & 0 \\
\star & \star & \star & \star & -\gamma W_{r}^{-1}
\end{array}\right]<0
$$




\section{Stability Robustness of the Work in [9]}

The work in [9] and [8] advocates only the optimization of antiwindup performance, that is, the minimization of the $\mathcal{L}_{2}$ gain of the operator $\mathcal{T}_{p}$. By setting $W_{r}=0$ and solving the LMI (23), we obtain a full-order compensator that only optimizes this performance. This approach is the continuous-time counterpart to the discrete-time approach of [9] . As argued in [8] and [9], this operator is central to obtaining desirable antiwindup behavior. It is interesting to examine whether this approach has any intrinsic robustness properties.

Suppose now that we consider output multiplicative uncertainty instead of additive uncertainty, that is, $\tilde{G}(s)=$ $\left(I+\Delta_{o}(s)\right) G_{2}(s)$, or, equivalently, that $W_{2}(s) \Delta_{G}(s)=$ $\Delta_{o}(s) G_{2}(s)=W_{3}(s) \tilde{\Delta}_{o}(s) G_{2}(s)$. Here, $W_{3}(s) \in \mathcal{R H}_{\infty}$ represents the "known" part of the output multiplicative uncertainty and $\tilde{\Delta}_{o} \in \boldsymbol{\Delta}$ represents the unknown but bounded part. ${ }^{2}$ In this case, our expression for $y_{\text {lin }}$ becomes

$$
y_{\text {lin }}=G_{1} d+G_{2} u_{\operatorname{lin}}+W_{3} \tilde{\Delta}_{o} G_{2}\left(u_{\operatorname{lin}}-M \mathcal{F}\left(u_{\text {lin }}\right)\right) .
$$

We are sure that the system is robustly stable when $\mathcal{F}\left(u_{\text {lin }}\right)=0$, as this is a property of the nominal linear system. Therefore, the smaller we can make the extra term $-W_{3} \tilde{\Delta}_{o} G_{2} M \mathcal{F}\left(u_{\text {lin }}\right)$, the closer to nominal robustness we shall be. We can do nothing about $\tilde{\Delta}_{O}$, so the logical approach is to make

$$
\left\|G_{2} M \mathcal{F}\left(u_{\text {lin }}\right)\right\|_{i, 2}=\left\|N \mathcal{F}\left(u_{\text {lin }}\right)\right\|_{i, 2} \geq\left\|G_{2}\right\|_{\infty}
$$

as small as possible, where the last inequality is a consequence of the $\mathcal{L}_{2}$ gain of a saturated system being bounded from below by that of the open-loop plant-see [19] and [8] for details. Note that as in [9], because $G_{2}(s)=N(s) M^{-1}(s)$ is a right coprime factorization of the plant $G_{2}(s)$, the quantity in (28) is exactly our performance operator norm $\left\|\mathcal{T}_{p}\right\|_{i, 2}$. Therefore the minimization of $\left\|\mathcal{T}_{p}\right\|_{i, 2}$ not only leads to desirable antiwindup performance but also endows the saturated system with some indirect robustness when the uncertainty is of the output multiplicative type. Note that the robustness is not guaranteed to approach that of the linear system and that multiplicative uncertainty is a narrower class of uncertainty than the additive type we have been considering heretofore.

Nevertheless, it does appear to explain some of the results of [22], where a discrete-time version of the results of [8] was implemented on a hard-disk system. In that work, few robustness problems were encountered, and the above analysis goes some way to explaining this.

\footnotetext{
${ }^{2}$ In terms of our additive uncertainty, observe that we now have two "known" parts either side of the unknown part, i.e., $W_{3}(s) \tilde{\Delta}_{o}(s) G_{2}(s)$, unless some of these transfer function matrices commute.
}

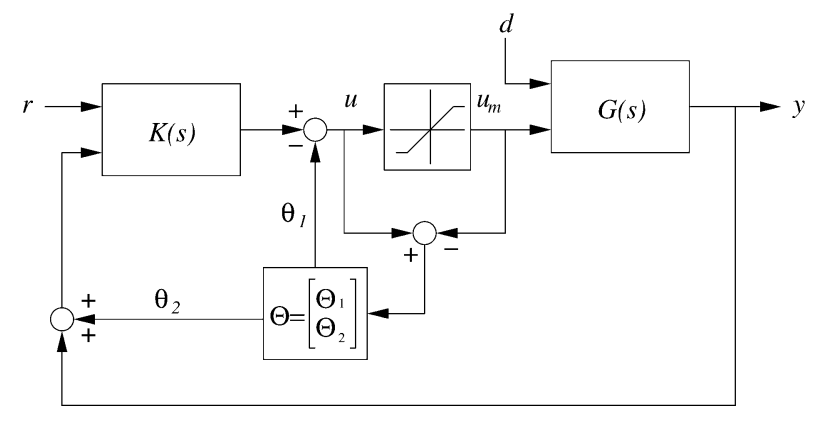

Fig. 7. Structure of static antiwindup scheme.

\section{RoBUST Static ANTIWINDUP SYNTHESIS}

Static antiwindup compensators are probably the most commonly used form of antiwindup compensator; they are simple and computationally efficient to implement. However, it is well known that static antiwindup compensators are not able to achieve global quadratic stability for all plant-controller combinations (see [19], for example). Thus, it is obvious that even if a static compensator existed for the nominal plant, without uncertainty present, there is no guarantee that one would exist for the uncertain plant. Therefore, it is more difficult to study the robust stability of static antiwindup compensators from the outset.

\section{A. A Decoupled Representation}

In line with the results presented earlier, to study the robustness of static antiwindup compensators, we first interpret them in the decoupled structure of [7]. Consider Fig. 7, where $\Theta=$ $\left[\begin{array}{ll}\Theta_{1}^{\prime} & \Theta_{2}^{\prime}\end{array}\right]^{\prime}$ is the static antiwindup compensator. Comparing this to Fig. 1, it follows that in order to realize this compensator in decoupled form, this is equivalent to choosing $M(s)$ [8] as

$$
M(s)=\left(I-K_{2}(s) G_{2}(s)\right)^{-1}\left(-K_{2}(s) G_{2}(s) \Theta_{2}+\Theta_{1}+I\right) .
$$

This is exactly the same approach taken in [8], where only performance was considered. Indeed, to combine performance objectives with robustness objectives, we need to consider Fig. 4 and attempt to find the $M(s)$ that minimizes $y_{d}$ and $z_{\Delta}$, in the same manner as before except with $M(s)$ restricted to the structure given in (29).

As in [8], one can find a realization of the key transfer functions to be considered as

$$
\left[\begin{array}{c}
M(s)-I \\
N(s) \\
M(s)
\end{array}\right] \sim\left\{\begin{array}{l}
\dot{\bar{x}}=\bar{A} \bar{x}+\left(B_{0}+\bar{B} \Theta\right) \tilde{u} \\
u_{d}=\bar{C}_{1} \bar{x}+\left(D_{01}+\bar{D}_{1} \Theta\right) \tilde{u} \\
y_{d}=\bar{C}_{2} \bar{x}+\left(D_{02}+\bar{D}_{2} \Theta\right) \tilde{u} \\
u_{\Delta}=\bar{C}_{1} \bar{x}+\left(\tilde{\Gamma}+\bar{D}_{1} \Theta\right) \tilde{u}
\end{array}\right.
$$

where the state-space matrices can be found in Appendix II.

$$
\left[\begin{array}{cccc}
Q A_{p}^{\prime}+A_{p} Q+L^{\prime} B_{p}^{\prime}+B_{p} L+\gamma^{-1} L^{\prime} W_{r} L & B_{p} U-L^{\prime}+\gamma^{-1} L^{\prime} W_{r} U & 0-\gamma^{-1} L^{\prime} W_{r} & Q C_{p}^{\prime}+L^{\prime} D_{p}^{\prime} \\
\star & -2 U+\gamma^{-1} U W_{r} U & I-\gamma^{-1} U W_{r} & U D_{p}^{\prime} \\
\star & \star & -\gamma I+\gamma^{-1} W_{r} & 0 \\
\star & \star & \star & -\gamma W_{p}^{-1}
\end{array}\right]
$$


In order to ensure that the antiwindup system in Fig. 4 is robust to uncertainty within the ball $\boldsymbol{\Delta}_{\gamma \delta}$, it is sufficient to ensure that (21) holds, where as before $W_{p}$ and $W_{r}$ are some positive definite weighting matrices and $z_{\Delta}=u_{\text {lin }}-u_{\Delta}$. Similarly to the previous section, this holds if the following inequality is satisfied:

$$
\begin{aligned}
& J=\frac{d}{d t} \bar{x}^{\prime} P \bar{x}+\left\|\begin{array}{c}
W_{p}^{\frac{1}{2}} y_{d} \\
W_{r}^{\frac{1}{2}} z_{\Delta}
\end{array}\right\|^{2}-\gamma^{2}\left\|u_{\operatorname{lin}}\right\|^{2} \\
& \quad+2 \tilde{u}^{\prime} W(u-\tilde{u})<0 \quad \forall\left[\begin{array}{lll}
\bar{x}^{\prime} & \tilde{u}^{\prime} & u_{\operatorname{lin}}^{\prime}
\end{array}\right]^{\prime} \neq 0 .
\end{aligned}
$$

After simplification, this inequality can be reposed as (32) as shown at the bottom of the page, which is an LMI in variables $Q>0$, diagonal $U>0, \gamma>0$, and matrix $L$. If this LMI is satisfied, a globally stabilizing compensator, ensuring the $\mathcal{L}_{2}$ gain bound (21) is satisfied, is given by $\Theta=L U^{-1}$. As before, this implies that the system in Fig. 4 is globally asymptotically stable for all $\Delta_{G} \in \boldsymbol{\Delta}_{\tilde{\gamma} \delta}$. Satisfaction of this inequality also guarantees well-posedness, although the details are fairly tedious [8].

\section{B. Local Static Results}

For arbitrary plant-controller combinations, the LMI (32) may not be feasible and hence a robust, globally quadratically stabilizing, static AW compensator will not be computable. However, there is a small relaxation that can be made to allow the synthesis of a locally stabilizing robust static AW compensator. We therefore modify our objectives and instead seek to ensure that our AW compensator bestows robust stability for all $\Delta_{G} \in \boldsymbol{\Delta}_{\tilde{\gamma} \delta}$, where

$$
\boldsymbol{\Delta}_{\tilde{\gamma} \delta}:=\left\{\Delta \in \mathcal{R H}_{\infty}: \quad\|\Delta\|_{\infty}<\frac{1}{\tilde{\gamma} \delta}\right\}
$$

providing that the magnitude of the control signal satisfies the bound

$$
\left|u_{i}(t)\right| \leq \beta_{i} \bar{u}_{i} \quad \forall i \quad \forall t \geq 0, \quad \beta_{i}>1
$$

This implies we are locally robust only providing our control signal remains sufficiently small, which can also be interpreted

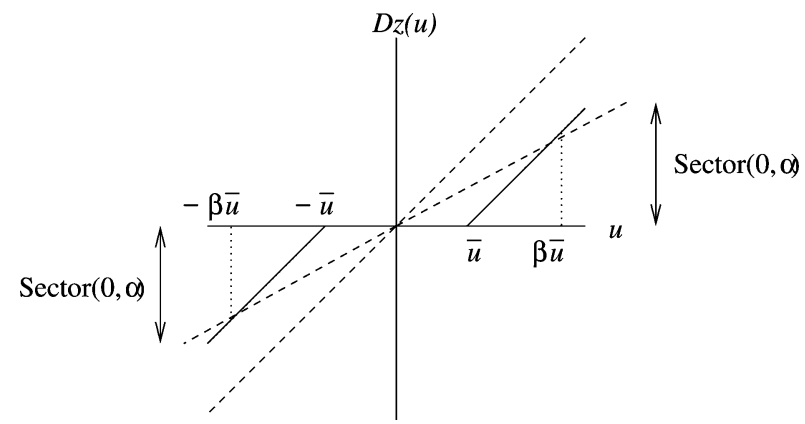

Fig. 8. Reduced sector bounds.

as the states of the system's remaining within some sufficiently small region of the state-space.

The approach we take is essentially the same as advocated in [23] (see also [24]), where we assume the dead-zone nonlinearity inhabits a smaller sector than the Sector $[0, I]$, namely, Sector $[0, \alpha I], \alpha \in(0,1)$. This can be interpreted as shown in Fig. 8. First consider the scalar case: notice that the standard dead zone inhabits Sector[0,1], as its graph lies between the gradient zero and gradient one line. However, if we assume that the control signal $u$ never exceeds a certain bound, as described by (34), it is evident that the graph of the dead zone lies within a narrower sector, namely, $\operatorname{Sector}[0, \alpha]$, where $\alpha=\beta /(\beta-1)$. For the multivariable case, we can follow a similar argument for all $i=\{1, \ldots, m\}$ elements of the dead zone and then define $\mathcal{A}:=\operatorname{diag}\left(\alpha_{1}, \ldots, \alpha_{m}\right)$. Thus, providing the components of $u$ are sufficiently small, i.e., $u_{i} \leq \beta_{i} \bar{u}_{i}=1 /\left(1-\alpha_{i}\right) \bar{u}_{i} \forall i$, then $\mathrm{Dz} \in \operatorname{Sector}[0, \mathcal{A}]$.

If we assume that $\mathrm{Dz} \in \operatorname{Sector}[0, \mathcal{A}]$ - which is equivalent to the bound (34) holding — then it follows [20] that the following inequality holds:

$$
\tilde{u}^{\prime} X\left[\mathcal{A}\left(u_{\text {lin }}-u_{d}\right)-\tilde{u}\right] \geq 0
$$

where again $X$ is some positive definite diagonal matrix.

Using this assumption, the synthesis LMI for a locally robust static AW compensator is given by (36) as shown at the bottom of the page, where $Q>0, U=\operatorname{diag}\left(\nu_{1}, \ldots, \nu_{m}\right)>0, L, \gamma>$

$$
\left[\begin{array}{ccccc}
Q \bar{A}^{\prime}+\bar{A} Q & B_{0} U+\bar{B} L-Q \bar{C}_{1}^{\prime} & 0 & Q \bar{C}_{2}^{\prime} & -Q \bar{C}_{1}^{\prime} \\
\star & -2 U-D_{01} U-\bar{D}_{1} L-U D_{01}^{\prime}-L^{\prime} \bar{D}_{1}^{\prime} & I & U D_{02}^{\prime}+L^{\prime} \bar{D}_{2}^{\prime} & -U \tilde{\Gamma}^{\prime}-L^{\prime} \bar{D}_{1}^{\prime} \\
\star & \star & -\gamma I & 0 & I \\
\star & \star & \star & -\gamma W_{p}^{-1} & 0 \\
\star & \star & \star & \star & -\gamma W_{r}^{-1}
\end{array}\right]<0
$$

$$
\left[\begin{array}{ccccc}
Q \bar{A}^{\prime}+\bar{A} Q & B_{0} U+\bar{B} L-Q \bar{C}_{1}^{\prime} \mathcal{A} & 0 & Q \bar{C}_{2}^{\prime} & -Q \bar{C}_{1}^{\prime} \\
\star & -2 U-\mathcal{A}\left(D_{01} U+\bar{D}_{1} L\right)+\left(U D_{01}^{\prime}+L^{\prime} \bar{D}_{1}^{\prime}\right) \mathcal{A} & \mathcal{A} & U D_{02}^{\prime}+L^{\prime} \bar{D}_{2}^{\prime} & -U \tilde{\Gamma}^{\prime}-L^{\prime} \bar{D}_{1}^{\prime} \\
\star & \star & -\gamma I & 0 & I \\
\star & \star & \star & -\gamma W_{p}^{-1} & 0 \\
\star & \star & \star & \star & -\gamma W_{r}^{-1}
\end{array}\right]<0
$$


0 are the LMI variables. If this LMI is satisfied, there exists a static AW compensator that ensures $\left\|\mathcal{T}_{p}\right\|_{i, 2}<\gamma / \sqrt{\underline{\sigma}\left(W_{p}\right)}$ and $\left\|\mathcal{T}_{r}\right\|_{i, 2}<\tilde{\gamma}=\gamma / \sqrt{\underline{\sigma}\left(W_{r}\right)}$, and therefore robust stability to all $\Delta_{G} \in \boldsymbol{\Delta}_{\tilde{\gamma} \delta}$ providing that the control signal $u(t)$ satisfied the bound (34). As before, we can recover this static AW compensator through $\Theta=L U^{-1}$.

Remark 3: The LMI (36) is useful in two respects. First, it allows the synthesis of a locally performing antiwindup compensator when a globally feasible one does not exist. Secondly, the additional free parameter $\mathcal{A}$, which controls the size of the sector where our results are valid, can be useful in robust synthesis, as it allows us to "trade off" robustness with sector size; often a more robust compensator can be designed at the expense of restricting the size of control input for which that robustness holds (that is, reducing the sector size). Note that $\mathcal{A}$ must be fixed for (36) to be an LMI.

Remark 4: It is even possible to go further and add another constraint to the LMI optimization (similar to, for example, [25] and [24]) to guarantee a region of the state-space for which asymptotic stability is guaranteed. Indeed, it can be proved (see Appendix III) that adding the $m$ LMI constraints (in $Q>0$, diagonal $U>0$, and $L$ ) as shown in (37) at the bottom of the page ensures that the dead zone stays within $\operatorname{Sector}[0, \mathcal{A}]$ for all $\bar{x} \in \mathcal{E}:=\left\{\bar{x}: \quad \bar{x}^{\prime} Q^{-1} \bar{x} \leq c_{\max }\right\}$, where $\tilde{c}_{\max }=1 / c_{\max }$. Thus one could choose to minimize

$$
\eta \gamma+(1-\eta) \tilde{c}_{\max } \quad \eta \in[0,1]
$$

subject to the LMI constraints (36) and (37) to allow performance and size region of attraction to be balanced in antiwindup compensator design. Ultimately, the LMIs (23) and (27) can be combined such that their simultaneous satisfaction ensures that the system in Fig. 4 is stable for all states of the nonlinear loop $\tilde{x} \in \mathcal{E}$ and for all $\Delta_{G} \in \Delta_{\tilde{\gamma} \delta}$.

Remark 5: In [26], a technique for antiwindup design was proposed based on a generalized sector condition, which in essence allows less conservative estimates of the region of attraction to be obtained. In principle, this generalized condition could replace (35), enabling the derivation of alternative LMIs to (23) and (27). Further work is needed to investigate this.

\section{EXAMPLE}

To demonstrate the implications of our results we use the academic example introduced in [27]. The example consists of a plant with a large resonant peak and the controller used is a two-degree-of-freedom controller with large feedback gain. However, we shall take this plant to be the perturbed plant, $\tilde{G}(s)$ rather than our nominal plant. We shall also use a controller with a slightly lower gain, for reasons which shall become clear later.

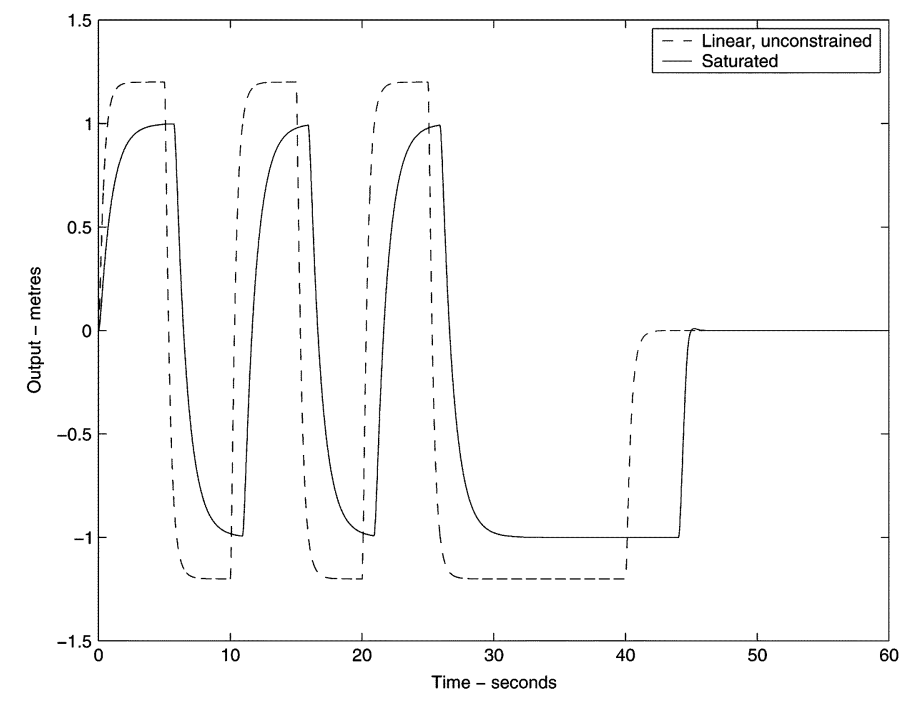

Fig. 9. Response of unperturbed system with no antiwindup.

\section{A. The Unperturbed System}

For our nominal plant we take the plant of [27] without the resonant peak (i.e., the system is critically damped). Thus, $G_{2}(s) \sim\left(A_{p}, B_{p}, C_{p}, D_{p}\right)$ is described by the state-space matrices

$$
\left[\begin{array}{l|l}
A_{p} & B_{p} \\
\hline C_{p} & D_{p}
\end{array}\right]=\left[\begin{array}{cc|c}
0 & 1 & 0 \\
-10 & -10 & 10 \\
\hline 1 & 0 & 0
\end{array}\right] .
$$

The linear controller $K(s)=\left[\begin{array}{ll}K_{1}(s) & K_{2}(s)\end{array}\right] \sim$ $\left(A_{c},\left[\begin{array}{ll}B_{c r} & B_{c}\end{array}\right], C_{c},\left[\begin{array}{ll}D_{c r} & D_{c}\end{array}\right]\right)$, which was designed for the plant $G(s)$, is described by the state-space matrices

$$
\begin{aligned}
{\left[\begin{array}{l|l|l}
A_{c} & B_{c r} & B_{c} \\
\hline C_{c} & D_{c r} & D_{c}
\end{array}\right] } \\
=\left[\begin{array}{ccc|cc}
-80 & 0 & 2.5 & 0 & -1 \\
1 & 0 & 0 & 0 & 0 \\
0 & 0 & -2.5 & 1 & 0 \\
\hline-9450 & 3375 & 337.5 & 0 & -135
\end{array}\right] .
\end{aligned}
$$

This is the same controller as in [27] but with a lower gain in the feedback loop. The dashed line in Fig. 9 shows the response of the linear system [i.e., the feedback interconnection of the plant (39) and the controller (40) without saturation] to a pulse reference of magnitude 1.2. As can be seen, the system is well behaved with no overshoot and a fast settling time. When the control input is saturated at \pm 1 , however, the system degrades to that shown by the solid line in Fig. 9. Although the system still exhibits no overshoot, the actuator saturation has impaired the system's ability to track its reference signal accurately and

$$
\left[\begin{array}{ccc}
Q & Q \bar{C}_{1}^{\prime} \mathcal{A} & Q \bar{C}_{1, i}^{\prime} \\
\star & 2 U+\mathcal{A}\left(D_{01} U+\bar{D}_{1} L\right)+\left(U D_{01}^{\prime}+L^{\prime} \bar{D}_{1}^{\prime}\right) \mathcal{A} & U D_{01, i}^{\prime}+L^{\prime} \bar{D}_{1, i}^{\prime} \\
\star & \star & \tilde{c}_{\max } \beta_{i}^{2} \bar{u}_{i}^{2}
\end{array}\right] \geq 0 \quad \forall i
$$




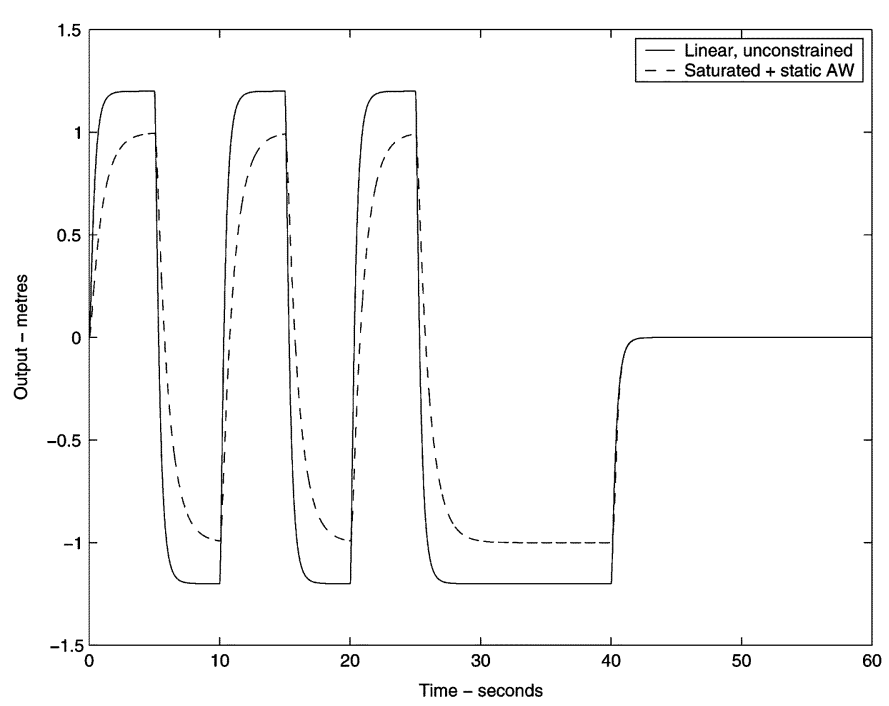

Fig. 10. Response of unperturbed system with optimal static antiwindup.

there appears to be a phase lag between the reference and the output.

To improve the behavior of the system, static antiwindup compensation as suggested in [8] is introduced. This antiwindup compensation minimizes the difference between the nominal linear system's response and the saturated system's response, i.e., $\left\|\mathcal{T}_{p}\right\|_{i, 2}$ only is minimized. The $\mathcal{L}_{2}$ gain of $\mathcal{T}_{p}, \gamma$ is calculated as $\gamma \approx 24$ and a static antiwindup compensator achieving this bound is computed as

$$
\Theta=\left[\begin{array}{c}
-0.1909 \\
0.1402
\end{array}\right]
$$

This compensator was implemented as shown in Fig. 7. Note that the unperturbed system is quadratically stabilizable by static antiwindup compensation, but we cannot be sure that the perturbed system also has this desirable property (in fact it does not). Fig. 10 shows the response of the system with static antiwindup [i.e., the feedback interconnection of of the plant (39) and the controller (40) with the AW compensator (41) and saturation] and it can be seen that the performance of the system has improved: the system output is now in phase with the reference demand, although the infeasibility of the reference means it is not possible for the output to track the input with the correct magnitude. The robust and full order antiwindup compensators introduced in the following sections both yield a similar response to that in Fig. 10 when used on the nominal system $G(s)$.

\section{B. The Perturbed System}

The true, or perturbed, plant $\tilde{G}(s)=G(s)+\Delta_{G}(s)$ is that given in [27]. This has a large resonant peak and is described by the following state-space matrices:

$$
\left[\begin{array}{c|c}
\tilde{A}_{p} & \tilde{B}_{p} \\
\hline \tilde{C}_{p} & \tilde{D}_{p}
\end{array}\right]=\left[\begin{array}{cc|c}
0 & 1 & 0 \\
-10 & -0.01 & 10 \\
\hline 1 & 0 & 0
\end{array}\right] .
$$

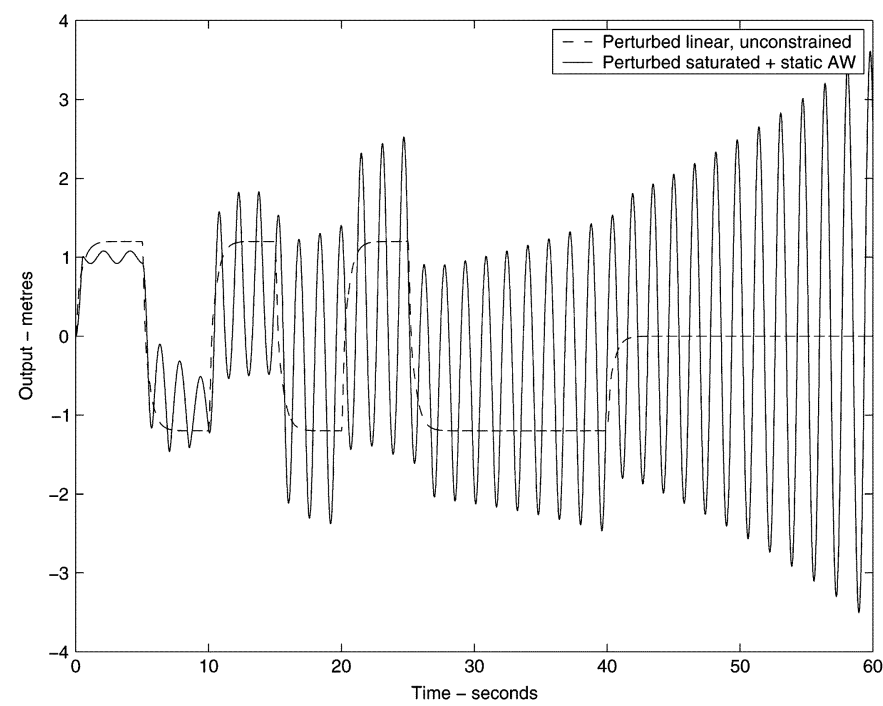

Fig. 11. Response of perturbed system with optimal static antiwindup.

The dashed line in Fig. 11 shows the response of this perturbed plant using the same controller as before and no input saturation [i.e., the feedback interconnection of the plant (42) and the controller (40) without saturation]; the controller yields a similar type of performance as before and hence can be considered satisfactory. However, when input saturation is introduced, the static antiwindup compensator (41), which was designed according to the method in [8] not considering uncertainty, actually drives the system unstable, as depicted by the solid line in Fig. 11. In fact, this static antiwindup is worse than no antiwindup at all (see Fig. 13), which at least remains stable, although significant phase lag and oscillations are observed. Note that for this perturbed plant and controller, most static antiwindup [28], [8], [19] methods ${ }^{3}$ are not feasible in a global sense, so we cannot expect it to stabilize the system in question for any reference input.

To overcome this problem, we choose $W_{p}=0.001$ and $W_{r}=$ 1 and synthesize a full order robust dynamic compensator according to the LMI (23). This yields the matrix $F$ as

$$
F=\left[\begin{array}{ll}
0.2242 & 0.0446
\end{array}\right] \times 10^{-4}, \quad \gamma \approx 1 .
$$

In this case, we have essentially the IMC solution, as $F$ is almost zero. As $\gamma \approx 1$, we can expect to recover the robustness results of the linear system. Indeed Fig. 12 shows the system's response with this AW compensator [i.e., the plant (42), the controller (40), and the compensator gain (43)] and we can see that the system is stable. Although the response is not as good as for the unperturbed system, it is substantially better than that of the static AW compensation. It should also be mentioned that this antiwindup compensator yields similar performance to the optimal static AW compensator when applied to the unperturbed plant; that is, the AW dynamic compensator designed using (43) and implemented on the unperturbed plant (39) gives similar performance to the compensator (41) implemented for the same unperturbed plant. In other words, the antiwindup compensator

\footnotetext{
${ }^{3}$ These methods all use quadratic Lyapunov functions and sector bounds. Static methods based on alternative Lyapunov functions or "sector bounds" may, however, be feasible.
} 


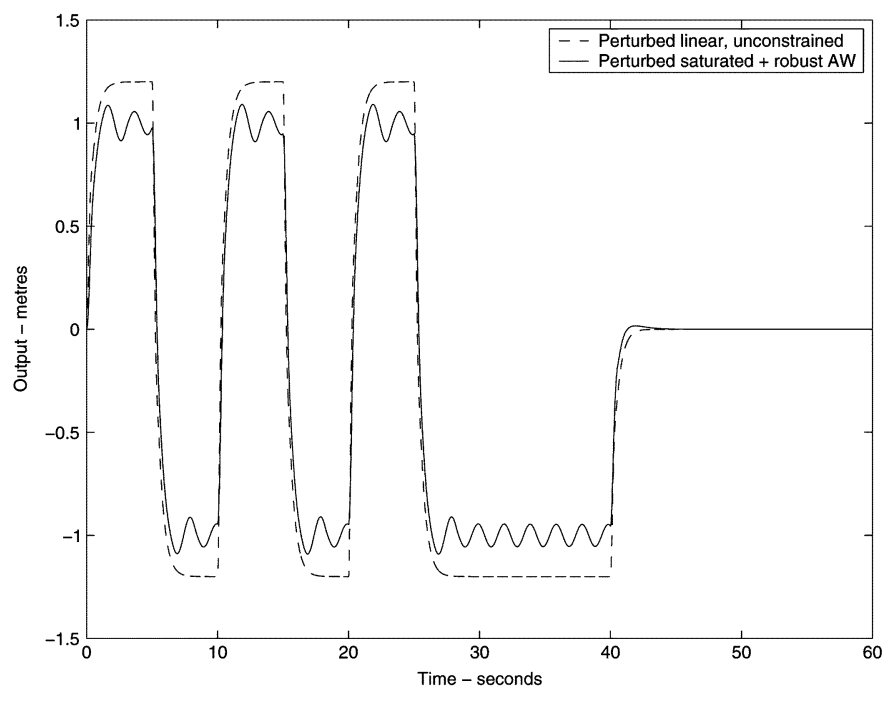

Fig. 12. Response of perturbed system with full-order robust antiwindup.

designed using (43) works well for both perturbed and unperturbed plants.

\section{A Robust Static Design}

The LMI (36) can be used to provide a static compensator that is guaranteed to be locally robust to perturbations to $G(s)$ providing the saturation level is sufficiently low, i.e., $u_{i}<\beta_{i} \bar{u}_{i} \forall i$, and hence, locally $\operatorname{Dz}(\cdot) \in \operatorname{Sector}[0, \mathcal{A}]$. Recall that because the perturbed plant $\tilde{G}(s)$ considered here is not globally quadratically stabilizable by static AW, this motivates the use of a local result instead. In this case, $\mathcal{A}=\alpha$ (single-input single-output system) and we found that many choices of the free parameter $\alpha \in(0,1)$ could enable us to synthesize a locally robust static antiwindup compensator. However, a value close to unity seemed to provide few robustness improvements, as essentially, we were close to global AW; thus although we could compute a static compensator, its large $\gamma$ value was indicative of poor robustness. Conversely, a value close to zero led to a nonlinear loop close to being ill-posed. A good compromise seemed to be $\alpha=0.6$, which guaranteed robustness provided that the control law did not exceed a magnitude of $1 /(1-\alpha)=2.5$. Using $\alpha=0.6$ with performance and robustness weights $W_{r}=1, W_{p}=0.5$, yielded the following static antiwindup compensator:

$$
\Theta=\left[\begin{array}{c}
-0.9689 \\
0.0001
\end{array}\right] \text {. }
$$

Fig. 13 shows a comparison between nominal responses, responses with uncertainty and no antiwindup, and the response with uncertainty and robust static antiwindup (all constrained). In contrast to the nonrobust static AW response (Fig. 11) for this input, reasonably good performance is obtained: although some oscillatory behavior is observed, the phase lag is minor (compared to no AW), and stability is maintained. However, the local nature of the results is revealed in Fig. 14, where a larger reference demand of magnitude 2.4 causes the system with the locally robust static AW to become unstable. Incidentally, for this reference demand, equipping the system with no

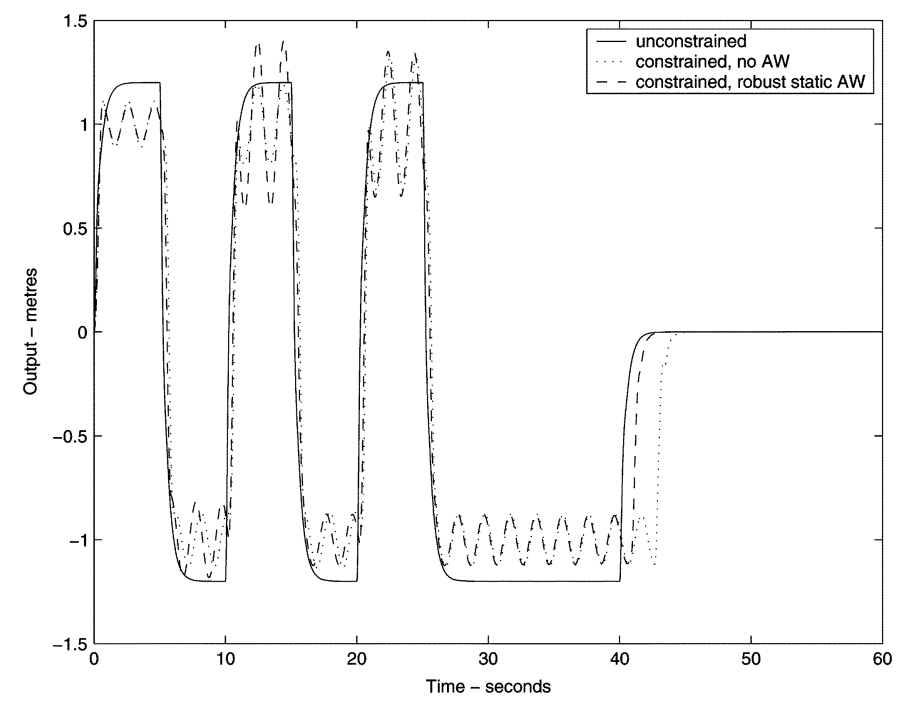

Fig. 13. Response of perturbed system with robust static AW.

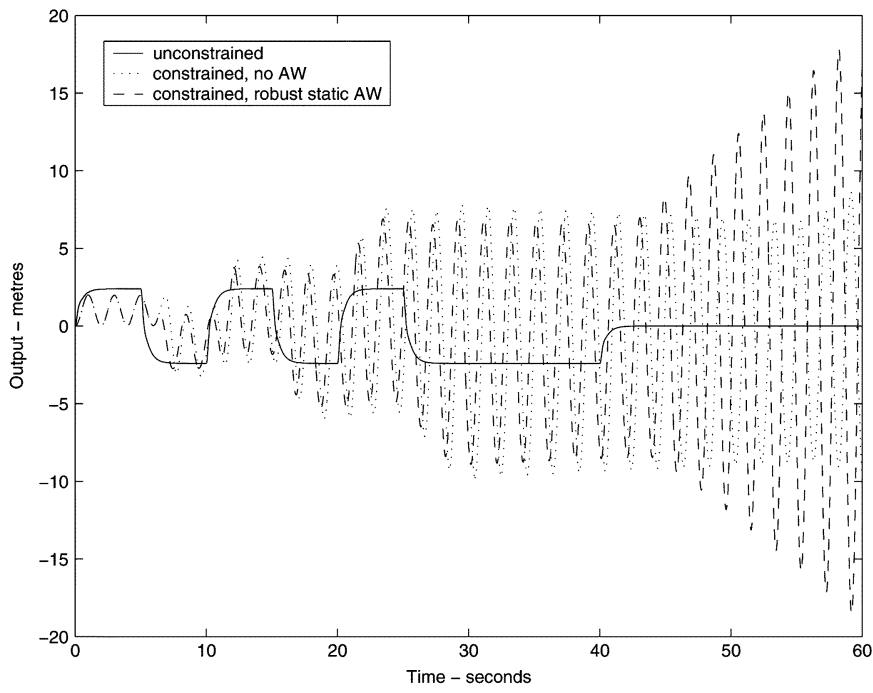

Fig. 14. Response of perturbed system with robust static AW: large reference demand.

antiwindup also causes instability. The conservatism of the local sector bound should also be mentioned: although in theory the local results only hold for $|u|<2.5$, in fact they appeared to hold for $|u|<4000$; only when this was exceeded (as in the case for a reference demand of 2.4) were stability problems encountered.

\section{Other Antiwindup Compensators}

As discussed in Section IV-D, the full-order antiwindup compensation method obtained by setting $W_{r}=0$ and solving the LMI (23) can, in a certain sense, provide a robust antiwindup solution. This type of antiwindup solution is the continuous time counterpart of the discrete-time full-order compensator described in [9]. We designed a full-order antiwindup compensator according to this approach, choosing $W_{p}=1$ and $W_{r}=0.0001 \approx 0$ and then solving the LMI (23). The optimal gain matrix $F$ was given by

$$
F=\left[\begin{array}{ll}
-1.3138 & -0.1424
\end{array}\right] \times 10^{4}, \quad \gamma \approx 1 .
$$




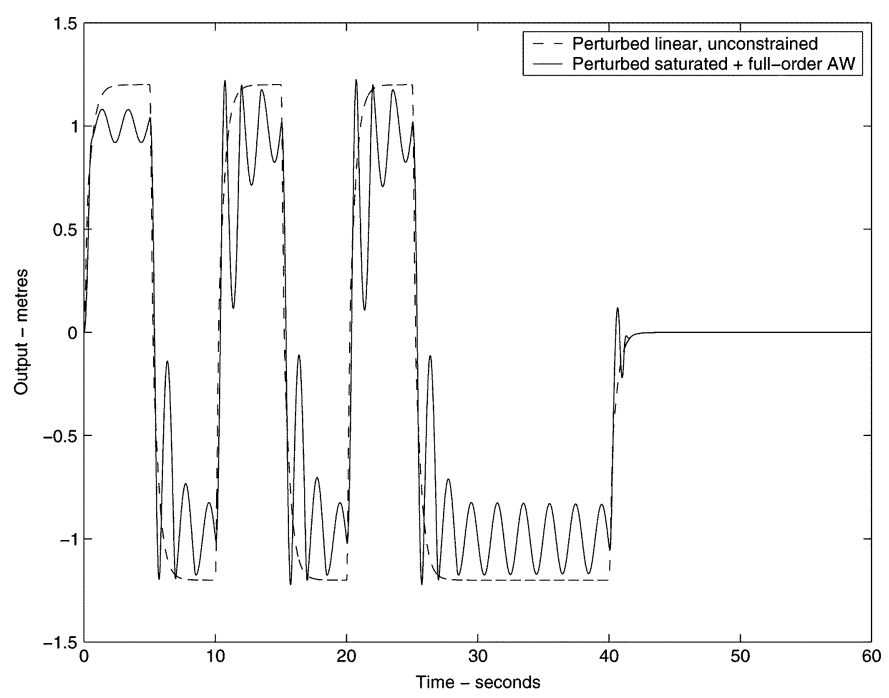

Fig. 15. Response of perturbed system with full-order antiwindup.

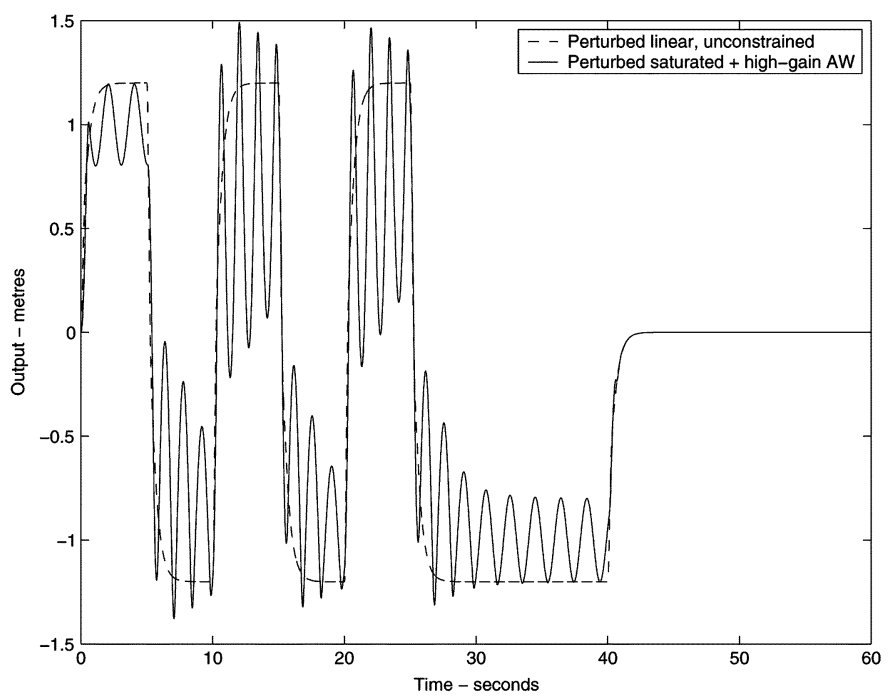

Fig. 16. Response of perturbed system with high-gain antiwindup.

It is important to realize that, in this case, the guaranteed robustness margin of the system is now given by $\gamma / \sqrt{W_{r}} \approx 100$, although this appears to be a conservative estimate. Again note that this places the poles of the AW compensator far into the left half-plane and, therefore, this would require a fast sampling frequency for correct implementation. Fig. 15 shows the response of the saturated system using this compensator. The stable response is indicative of the scheme's intrinsic robustness properties, although it does appear to be more oscillatory than that of the robust antiwindup compensator.

For purposes of comparison and due to its industrial popularity, we also tested a high-gain antiwindup compensator [13], [14]. In terms of Fig. 7, we chose $\Theta=\left[\begin{array}{ll}0 & 14\end{array}\right]^{\prime}$. No formal performance or stability guarantees accompany this scheme. Nevertheless the response of the unperturbed system using this antiwindup scheme is very similar to the response with the optimal static scheme (Fig. 10). When this compensator was tested on the perturbed plant (Fig. 16) stability was also obtained, although we note the very oscillatory response, which may be indicative of poor robustness. Its response was certainly not as good as that of the robust antiwindup compensator shown in Fig. 12, although it was obviously superior to that of the optimal static scheme shown in Fig. 11.

\section{CONCLUSION}

This paper has introduced a framework for synthesizing robust antiwindup compensators for open-loop plants subject to additive uncertainties. The problem was posed in a similar way to that of linear $\mathcal{H}_{\infty}$ control theory, and its solution appears as a set of LMIs of a similar type to those proposed in [8]. The attractive feature of the proposed solution is that the class of uncertainties considered are those that are routinely used by control practitioners, which underlines the practical relevance of the results. As an important aside, we have also demonstrated the optimal robustness of the much-denigrated IMC antiwindup strategy.

We note that many of the simple antiwindup schemes also seem to be quite robust in practice. For example, the Hanus scheme [12], which is a particular type of static AW compensator, has been the practitioners' method of choice for some time ([14] and [29] contain examples). Using the static results contained in this paper, it is possible to analyze the robustness of such a scheme, albeit typically locally. As the results here use the framework of [7], in which most linear AW schemes can be interpreted, the robustness results here should find wide applicability.

Finally, it should be mentioned that recently the so-called weakened antiwindup scheme has been introduced by Galeani et al. (see [30]), which promises to offer improved robustness to saturated systems but at the expense of slightly adjusting the linear loop. This should reveal further avenues of research.

\section{APPENDIX I \\ ProOf That LiNEAR RoBUSTNESS IS OPTIMAL}

In order to prove that linear robustness is optimal, we need to prove that the induced norm of the nonlinear operator $\mathcal{T}_{r}$ : $u_{\text {lin }} \mapsto z_{\Delta}$ can never be less than unity. i.e.,

$$
\left\|\mathcal{T}_{r}\right\|_{i, 2}=\sup _{0 \neq u_{\text {lin }} \in \mathcal{L}_{2}} \frac{\left\|u_{\text {lin }}-M \mathcal{F}\left(u_{\text {lin }}\right)\right\|_{2}}{\left\|u_{\text {lin }}\right\|_{2}} \geq 1
$$

To see that this is the case, assume the opposite: $\left\|\mathcal{T}_{r}\right\|_{i, 2}<1$. Now choose a signal $u_{\text {lin }}(t) \in \mathcal{L}_{2}$ such that $u_{\text {lin }}(t) \preceq \bar{u} \forall t$; such a signal always exists for $\bar{u}_{i}>0$. Then it follows that $\mathcal{F}\left(u_{\text {lin }}\right)=0 \forall t$ and thus that

$$
\left\|\mathcal{T}_{r}\right\|_{i, 2}=\sup _{0 \neq u_{\text {lin }} \in \mathcal{L}_{2}} \frac{\left\|u_{\text {lin }}\right\|_{2}}{\left\|u_{\text {lin }}\right\|_{2}}=1 .
$$

Thus we have a contradiction and hence $\left\|\mathcal{T}_{r}\right\|_{i, 2} \geq 1$.

\section{APPENDIX II}

StATE-SPACE MATRICES FOR RobUSt StATIC ANTIWINDUP

Given a controller $K(s)=\left[K_{1}(s) K_{2}(s)\right] \sim$ $\left(A_{c},\left[\begin{array}{ll}B_{c r} & B c\end{array}\right], C_{c},\left[\begin{array}{ll}D_{c r} & D_{c}\end{array}\right]\right)$ and plant $G_{2}(s) \sim$ 


$$
\begin{aligned}
& {\left[\begin{array}{l}
\bar{x} \\
\tilde{u}
\end{array}\right]^{\prime}\left[\begin{array}{cc}
\bar{C}_{1, i}^{\prime} \bar{C}_{1, i} & \bar{C}_{1, i}^{\prime}\left(D_{01}+\bar{D}_{1} \Theta\right)_{i} \\
\left(D_{01}+\bar{D}_{1} \Theta\right)_{i}^{\prime} \bar{C}_{1, i} & \left(D_{01}+\bar{D}_{1} \Theta\right)_{i}^{\prime}\left(D_{01}+\bar{D}_{1} \Theta\right)_{i}
\end{array}\right]\left[\begin{array}{l}
\bar{x} \\
\tilde{u}
\end{array}\right] \leq \beta_{i}^{2} \bar{u}_{i}^{2} \quad \forall i} \\
& {\left[\begin{array}{l}
\bar{x} \\
\tilde{u}
\end{array}\right]^{\prime}\left[\begin{array}{cc}
\bar{C}_{1, i}^{\prime} \bar{C}_{1, i} & \bar{C}_{1, i}^{\prime}\left(D_{01}+\bar{D}_{1} \Theta\right)_{i} \\
\left(D_{01}+\bar{D}_{1} \Theta\right)_{i}^{\prime} \bar{C}_{1, i} & \left(D_{01}+\bar{D}_{1} \Theta\right)_{i}^{\prime}\left(D_{01}+\bar{D}_{1} \Theta\right)_{i}
\end{array}\right]\left[\begin{array}{l}
\bar{x} \\
\tilde{u}
\end{array}\right] \leq \beta_{i}^{2} \bar{u}_{i}^{2} \bar{x}^{\prime} P \bar{x} / c_{\max } \quad \forall i} \\
& \bar{x}^{\prime} P \bar{x}-\frac{c_{\max }}{\beta_{i}^{2} \bar{u}_{i}^{2}}\left[\begin{array}{c}
\bar{x} \\
\tilde{u}
\end{array}\right]^{\prime}\left[\begin{array}{cc}
\bar{C}_{1, i}^{\prime} \bar{C}_{1, i} & \bar{C}_{1, i}^{\prime}\left(D_{01}+\bar{D}_{1} \Theta\right)_{i} \\
\left(D_{01}+\bar{D}_{1} \Theta\right)_{i}^{\prime} \bar{C}_{1, i} & \left(D_{01}+\bar{D}_{1} \Theta\right)_{i}^{\prime}\left(D_{01}+\bar{D}_{1} \Theta\right)_{i}
\end{array}\right]\left[\begin{array}{l}
\bar{x} \\
\tilde{u}
\end{array}\right] \geq 0 \quad \forall i \\
& {\left[\begin{array}{ccc}
P & \bar{C}_{1}^{\prime} \mathcal{A} X & \bar{C}_{1, i}^{\prime} \\
\star & 2 X+X \mathcal{A}\left(D_{01}+\bar{D}_{1} \Theta\right)+\left(D_{01}+\bar{D}_{1} \Theta\right)^{\prime} \mathcal{A} X & \left(D_{01}+\bar{D}_{1} \Theta\right)_{i}^{\prime} \\
\star & \star & \beta_{i}^{2} \bar{u}_{i}^{2} / c_{\max }
\end{array}\right] \geq 0 \quad \forall i}
\end{aligned}
$$

$\left(A_{p}, B_{p}, C_{p}, D_{p}\right)$, the state-space matrices for the representation given in (30) are given by

$$
\begin{aligned}
\bar{A} & :=\left[\begin{array}{cc}
A_{p}+B_{p} \tilde{\Gamma} D_{c} C_{p} & B_{p} \tilde{\Gamma} C_{c} \\
B_{c} \Gamma C_{p} & A_{c}+B_{c} \Gamma D_{p} C_{c}
\end{array}\right] \\
B_{0} & :=\left[\begin{array}{c}
B_{p} \tilde{\Gamma} \\
B_{c} \Gamma D_{p}
\end{array}\right], \quad \bar{B}:=\left[\begin{array}{cc}
B_{p} \tilde{\Gamma} & -B_{p} \tilde{\Gamma} D_{c} \\
B_{c} \Gamma D_{p} & -B_{c} \Gamma
\end{array}\right] \\
\Theta & :=\left[\begin{array}{c}
\Theta_{1} \\
\Theta_{2}
\end{array}\right] \\
\bar{C}_{1} & :=\left[\begin{array}{ll}
\tilde{\Gamma} D_{c} C_{p} & \tilde{\Gamma} C_{c}
\end{array}\right], \quad D_{01}:=\tilde{\Gamma} D_{c} D_{p} \\
\bar{D}_{1} & :=\left[\begin{array}{ll}
I+\tilde{\Gamma} D_{c} D_{p} & -\tilde{\Gamma} D_{c}
\end{array}\right] \\
\bar{C}_{2} & :=\left[\begin{array}{ll}
\Gamma C_{p} & \Gamma D_{p} C_{c}
\end{array}\right], \quad D_{02}:=\Gamma D_{p} \\
\bar{D}_{2} & :=\left[\begin{array}{ll}
\Gamma D_{p} & -\Gamma D_{p} D_{c}
\end{array}\right]
\end{aligned}
$$

where $\Gamma:=\left(I-D_{p} D_{c}\right)^{-1}$ and $\tilde{\Gamma}:=\left(I-D_{c} D_{p}\right)^{-1}$.

\section{APPENDIX III}

\section{GUARANTEEING A REGION OF ASYMPTOTIC STABILITY}

Essentially the ideas follow from [23] and [25] or [24], the main difference being that we consider loops with direct feedthrough terms. First assume that $\tilde{x} \in \mathcal{E}$ is small enough such that $\operatorname{Dz}(\cdot) \in \operatorname{Sector}[0, \mathcal{A}]$. This implies

$$
\left|u_{i}\right|=\left|\bar{C}_{1} \bar{x}+\left(D_{01}+\bar{D}_{1} \Theta\right) \tilde{u}\right|_{i} \leq \beta_{i} \bar{u}_{i} \quad \forall i
$$

which is equivalent to (51). Again, because $\bar{x} \in \mathcal{E}$, it follows that

$$
\bar{x}^{\prime} P \bar{x} / c_{\max } \leq 1 \quad \forall x \in \mathcal{E} .
$$

Thus it follows that (51) holds for all $\bar{x} \in \mathcal{E}$ if (52) holds, which after rearrangement is equivalent to (53). Rewriting the sector condition as

$$
2 \tilde{u}^{\prime} X\left[\tilde{u}+\mathcal{A} \bar{C}_{1} \bar{x}+\mathcal{A}\left(D_{01}+\bar{D}_{1} \Theta\right) \tilde{u}\right] \leq 0
$$

and using the S-procedure, a sufficient condition for (53) to hold (and therefore for (51) to hold) is, after applying the Schur complement, obtained as shown in (51)-(54) at the top of the page. Using the congruence transformation $\operatorname{diag}\left(P^{-1}, X^{-1}, I\right)=\operatorname{diag}(Q, U, I)$, we arrive at (37).

\section{REFERENCES}

[1] S. Tarbouriech and G. Garcia, Control of Uncertain Systems with Bounded Inputs. London, U.K.: Springer-Verlag, 1997.

[2] M. Turner, G. Herrmann, and I. Postlethwaite, "Accounting for uncertainty in anti-windup synthesis," in Proc. Amer. Contr. Conf., 2004.

[3] M. Turner and I. Postlethwaite, "Robustness analysis of general linear conditioning schemes," in Proc. Workshop Syst. Time Domain Constraints, Eindhoven, The Netherlands, 2000.

[4] A. Teel and N. Kapoor, "The $\mathcal{L}_{2}$ anti-windup problem: Its definition and solution," in Proc. Eur. Contr. Conf., 1997.

[5] K. Zhou, J. Doyle, and K. Glover, Robust and Optimal Control. Englewood Cliffs: Prentice-Hall, 1996.

[6] P. Weston and I. Postlethwaite, "Analysis and design of linear conditioning schemes for systems containing saturating actuators," in Proc. IFAC Nonlinear Contr. Syst. Design Symp., 1998.

[7] P. Weston and I. Postlethwaite, "Linear conditioning for systems containing saturating actuators," Automatica, vol. 36, no. 9, pp. 1347-1354, 2000.

[8] M. Turner and I. Postlethwaite, "A new perspective on static and low order anti-windup synthesis," Int. J. Contr., vol. 77, no. 1, pp. 27-44, 2004.

[9] M. Turner, G. Herrmann, and I. Postlethwaite, "Discrete time antiwindup-Part 1: Stability and performance," in Proc. Eur. Contr. Conf., 2003.

[10] G. Herrmann, M. Turner, and I. Postlethwaite, "Discrete time antiwind-up-Part 2: Extension to sampled data case," in Proc. Eur. Contr. Conf., 2003.

[11] G. Herrmann, M. Turner, and I. Postlethwaite, "Discrete-time and sampled-data anti-windup synthesis: Stability and performance," Int. J. Syst. Sci., 2006.

[12] R. Hanus, M. Kinnaert, and J. Henrotte, "Conditioning technique, a general anti-windup and bumpless transfer method," Automatica, vol. 23, no. 6, pp. 729-739, 1987.

[13] C. Edwards and I. Postlethwaite, "Anti-windup and bumpless transfer schemes," Automatica, vol. 34, no. 2, pp. 199-210, 1998.

[14] R. Hyde, "The application of robust control to Vstol aircraft," Ph.D. dissertation, Dept. of Eng., Univ. of Cambridge, Cambridge, U.K., 1991.

[15] A. Zheng and M. Morari, "Anti-windup using internal model control," Int. J. Contr., 1994.

[16] G. Grimm, I. Postlethwaite, A. Teel, M. Turner, and L. Zaccarian, "Case studies using linear matrix inequalities for optimal anti-windup synthesis," Eur. J. Contr., vol. 9, pp. 463-473, 2003.

[17] J. Akkermans, A. Will, and W. Heath, "Robust cross-directional control of paper making machines with saturating actuators," in Proc. Contr. Syst. 2004, 2004.

[18] M. Kothare, P. Campo, M. Morari, and C. Nett, "A unified framework for the study of anti-windup designs," Automatica, vol. 30, no. 12, pp. $1869-1883,1994$.

[19] G. Grimm, J. Hatfield, I. Postlethwaite, A. Teel, M. Turner, and L. Zaccarian, "Anti-windup for stable linear systems with input saturation: An LMI based synthesis," IEEE Trans. Autom. Control, vol. 48, no. 9, pp. 1509-1525, 2003. 
[20] H. Khalil, Nonlinear Systems. Englewood Cliffs, NJ: Prentice-Hall, 1996.

[21] S. Skogestad and I. Postlethwaite, Multivariable Feedback Control: Analysis and Design, 2nd ed. Chichester, U.K.: Wiley, 2005.

[22] G. Herrmann, M. Turner, and I. Postlethwaite, "Practical implementation of a novel anti-windup scheme in a HDD-dual-stage servo-system," IEEE/ASME Trans. Mechatronics, vol. 9, no. 3, pp. 580-592, 2004.

[23] H. Hindi and S. Boyd, "Analysis of linear systems with saturation using convex optimization," in Proc. Amer. Contr. Conf., 1998.

[24] T. Kiyama and K. Sawada, "LMI-based $\mathcal{L}_{2}$ analysis/synthesis with saturating control via generalized sector approach," in Proc. IFAC World Congr., 2005.

[25] S. Tarbouriech and J. G. da Silva Jr, "Synthesis of controllers for continuous-time delay systems with saturating controls via LMI's," IEEE Trans. Autom. Control, vol. 45, no. 1, pp. 105-111, 2000.

[26] J. G. da Silva Jr and S. Tarbouriech, "Anti-windup design with guaranteed regions of stability: An LMI-based approach," IEEE Trans. Autom. Control, vol. 50, no. 1, pp. 106-111, 2005.

[27] L. Zaccarian and A. Teel, "A common framework for anti-windup, bumpless transfer and reliable designs," Automatica, vol. 38, pp. 1735-1744, 2002.

[28] E. Mulder, M. Kothare, and M. Morari, "Multivariable anti-windup controller synthesis using linear matrix inequalities," Automatica, vol. 37, pp. 1407-1416, 2001.

[29] I. Postlethwaite, R. Samar, B. Choi, and D. Gu, "A digital multimode $\mathcal{H}_{\infty}$ controller for the spey turbofan engine," in Proc. Eur. Contr. Conf., 1995, pp. 3881-286.

[30] S. Galeani, S. Nicosa, A. Teel, and L. Zaccarian, "Output feedback compensators for weakened anti-windup of additively perturbed systems," in Proc. IFAC World Congr., 2005.

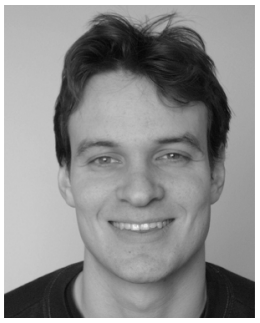

Matthew C. Turner was born in Corby, U.K., in 1975. He received the B.Eng. degree in electrical and electronic engineering from the University of Surrey, U.K., in 1996 and the Ph.D. degree in control engineering from the University of Leicester, U.K., in 2000 .

His main research interests are centered around robust control, flight control, and the control of systems containing isolated nonlinearities. He has designed and implemented robust controllers and antiwindup compensators on several industrial engineering systems. He is currently involved with the European aerospace group GARTEUR in investigating the phenomenon of pilot-induced oscillations.

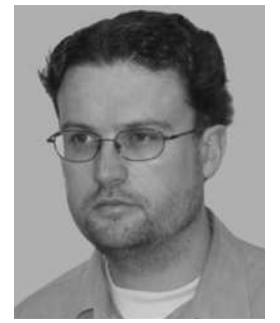

Guido Herrmann (M'99-SM'06) was born in Berlin, Germany, in 1972. He received the Diplom-Ingenieur der Elektrotechnik degree (with highest honors) from the Technische Universitaet zu Berlin, Germany, in 1997 and the Ph.D. degree from the University of Leicester, U.K., in 2001.

His doctoral work was supported by the Daimler-Benz-Foundation/Germany and later by a Marie-Curie Fellowship. From 2001 to 2003, he was a Senior Research Fellow with A*STAR Data Storage Institute, Singapore. From 2003 until 2005, he was a Research Associate with the University of Leicester followed by a one-year secondment as a Lecturer. He joined the University of Bristol, U.K., as a Lecturer in dynamics in March 2007. His research interests are in the theory and application of nonlinear and robust control, in particular sampled data, antiwindup, sliding mode, and $\mathrm{NN}$-control as well as regulation of chaotic systems.

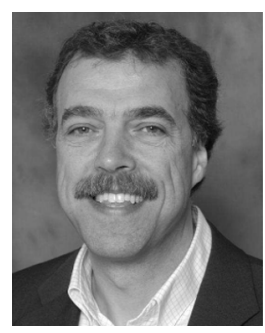

Ian Postlethwaite (M'75-SM'92-F'01) was born in Wigan, U.K., in 1953. He received the B.Sc. (Eng.) degree from Imperial College, London University, U.K., in 1975 and the Ph.D. degree from St. Catharine's College, Cambridge University, U.K., in 1978.

From 1978 to 1981, he was a Research Fellow with Trinity Hall, Cambridge University, and spent six months with General Electric Company, Schenectady, NY. In 1981, he received a Lectureship in engineering science at Oxford University, U.K., and a Tutorial Fellowship with Oriel College. In 1988, he became a Chair of Engineering at the University of Leicester, where he was Head of the department from 1995 to 2004. At Leicester, he is currently Head of the Control and Instrumentation Research Group and a Pro-Vice-Chancellor. He has held visiting positions at the University of California at Berkeley and at the Australian National University. His research involves theoretical contributions to the field of robust multivariable control and the application of advanced control system design to engineering systems. He is a coauthor (with S. Skogestad) of Multivariable Feedback Control (New York: Wiley, 1996 and 2005). He has received support for his research from EPSRC (including two platform grants), the European Union, government, and industry.

Dr. Postlethwaite is a Fellow of the Royal Academy of Engineering, the Institute of Electrical and Electronics Engineers, the Institution of Electrical Engineers, and the Institute of Measurement and Control. 